ARTICLE

https://doi.org/10.1038/s41467-020-14396-9

\title{
Sexual-dimorphism in human immune system aging
}

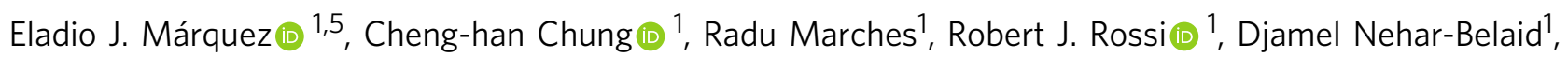
Alper Eroglu (1) 1, David J. Mellert (1) 1, George A. Kuchel (1) 2,6, Jacques Banchereau (i) 1,6 \& Duygu Ucar (1) 1,3,4,6*

Differences in immune function and responses contribute to health- and life-span disparities between sexes. However, the role of sex in immune system aging is not well understood. Here, we characterize peripheral blood mononuclear cells from 172 healthy adults 22-93 years of age using ATAC-seq, RNA-seq, and flow cytometry. These data reveal a shared epigenomic signature of aging including declining naïve $T$ cell and increasing monocyte and cytotoxic cell functions. These changes are greater in magnitude in men and accompanied by a male-specific decline in B-cell specific loci. Age-related epigenomic changes first spike around late-thirties with similar timing and magnitude between sexes, whereas the second spike is earlier and stronger in men. Unexpectedly, genomic differences between sexes increase after age 65, with men having higher innate and pro-inflammatory activity and lower adaptive activity. Impact of age and sex on immune phenotypes can be visualized at https:// immune-aging.jax.org to provide insights into future studies.

\footnotetext{
${ }^{1}$ The Jackson Laboratory for Genomic Medicine, Farmington, CT 06032, USA. ${ }^{2}$ University of Connecticut Center on Aging, UConn Health Center, Farmington, CT 06030, USA. ${ }^{3}$ Institute of Systems Genomics, University of Connecticut, Farmington, CT 06032, USA. ${ }^{4}$ Department of Genetics and Genome Sciences, University of Connecticut Health Center, Farmington, CT 06030, USA. ${ }^{5}$ Present address: Sanofi US, Cambridge, MA 02139, USA. ${ }^{6}$ These authors contributed equally: George A. Kuchel, Jacques Banchereau, Duygu Ucar. *email: duygu.ucar@jax.org
} 
$\mathrm{H}$ uman peripheral blood mononuclear cells (PBMCs) undergo both cell-intrinsic and cell-compositional changes (i.e., cell frequencies) with age, where certain immune functions are impaired and others are remodeled ${ }^{1}$. Analyses of human blood samples uncovered significant aging-related changes in gene expression ${ }^{2}$ and DNA methylation levels ${ }^{3}$. Recent studies revealed that chromatin accessibility of purified immune cells, especially $\mathrm{CD} 8^{+} \mathrm{T}$ cells, change significantly with aging, impacting the activity of important receptor molecules, signaling pathways, and transcription factors $(\mathrm{TF})^{4,5}$. Together, these changes likely contribute to aging-related immunodeficiency and ultimately to increased frequency of morbidity and mortality among older adults. However, it is unclear to what extent these aging-associated changes are shared between men and women.

Immune systems of men and women function and respond to infections and vaccination differently ${ }^{6}$. For example, $80 \%$ of autoimmune diseases occur in women, who typically show stronger immune responses than males ${ }^{7}$. Stronger responses in women produce faster pathogen clearance and better vaccine responsiveness, but also contribute to increased susceptibility to inflammatory and auto-immune diseases. Although not systematically described, these differences likely stem from differences in both cell frequencies and cell-intrinsic programs. For example, a study in young individuals showed that women have more B cells (percentage and absolute cell counts) in their blood than men ${ }^{8}$. Moreover, hundreds of genes are differentially expressed between young men and young women in sorted B cells ${ }^{9}$. Recently, sexbiased transcripts have been comprehensively described in purified immune cells $(n=1800)$, the majority of which are autosomal ${ }^{10}$. Furthermore, age- and sex-dependent differences in immune responses to stimuli have been observed ${ }^{11,12}$. Despite the importance of sex and age in shaping immune cell functions and responses, it is not known whether men's and women's immune systems go through similar changes throughout their lifespan, and whether these changes occur at the same time and at the same rate.

To study this, we profiled PBMCs of healthy adults by carefully matching the ages of male and female donors. Computational pipelines for functional enrichments, trajectory and breakpoint analyses revealed immune system aging signatures including longitudinal genomic trends. These findings uncovered in which ways aging differentially affects the immune systems of men and women. These results are shared via a searchable $\mathrm{R}$ Shiny application (https://immune-aging.jax.org/).

\section{Results}

Profiling PBMCs of healthy adults. We recruited 172 communitydwelling healthy volunteers (91 women, 81 men) whose ages span 22-93 years old (Fig. 1a, Supplementary Table 1): 54 young (ages 22-40: 23 men, 31 women), 59 middle-aged (ages 41-64: 31 men, 28 women), and 59 older subjects (65+: 27 men, 32 women). No significant differences were detected between sexes in their frailty scores or age distributions (Supplementary Fig. 1g, Supplementary Table 1). PBMCs were profiled using ATAC-seq (54 men, 66 women), RNA-seq (41 men, 34 women), and flow cytometry (62 men, 67 women). Male and female samples for each assay were comparable in terms of frailty scores, BMI, and age except for young samples profiled with flow cytometry; young women were slightly older than men ( 32.3 vs. $\sim 28.35)$ ( $t$-test $p$-value $=0.05)$ (Supplementary Table 1$)$. We took this difference into consideration while interpreting the flow cytometry data.

Aging is the main driver of variation in PBMC ATAC-seq and RNA-seq data. To identify major sources of variation in genomics data, we conducted principal component analyses (PCA) using open chromatin regions (OCRs; $n=78,167$ ) and expressed genes $(n=12,350)$ from high-quality samples (100 ATAC-seq, 74 RNAseq) (Supplementary Table 2). The first principal component (PC1) captured $15 \%$ of the variation in ATAC-seq and $18.6 \%$ in RNA-seq data and associated to age groups (Fig. 1b, Supplementary Fig. 1a, b, Supplementary Table 3). PC1 differences between young and old samples were more significant in men (ATAC-seq: $p<0.05$ vs. $p<0.001$, RNA-seq: $p=$ N.S. vs. $p=0.03$, Wilcoxon rank-sum test) (Fig. 1b). Annotation of PC1-related genes using immune modules ${ }^{13}$ revealed genomic declines associated with the 'T cell' module and gains associated with 'myeloid lineage' and 'inflammation' (Fig. 1c), confirmed by enrichment analyses based on single-cell RNA-seq data (Supplementary Fig. 1c). Together, these results suggest that aging is the main driver of variation in PBMC epigenomes/transcriptomes, where age-related variation is negatively associated with naive $\mathrm{T}$ cells and positively associated with myeloid lineage and inflammation, in agreement with the signatures of immunosenescence including impaired responsiveness of adaptive immunity and increases in low-grade and systemic inflammation (i.e., inflamm-aging) with age, which together contribute to aging diseases $^{14}$.

Aging-related changes in PBMC cell compositions. We quantified the frequencies of $\mathrm{CD} 4^{+}$and $\mathrm{CD} 8^{+} \mathrm{T}$ cells, $\mathrm{CD} 19^{+} \mathrm{B}$ cells, and $\mathrm{CD} 14^{+}$monocytes in PBMCs (67 women, 62 men) (Supplementary Table 4, Supplementary Fig. 2a). Among these, the proportions of $\mathrm{CD}^{+} \mathrm{T}$ cells were the most significantly affected with age ( $p=8.2 \mathrm{e}^{-07}$ for men, $p=0.0069$ for women, Wilcoxon) (Fig. 1d). Similarly, CD $4^{+} \mathrm{T}$ cell frequencies declined both in men (Wilcoxon $p=0.01$ ) and women (Wilcoxon $p=0.0026$ ). However, we detected a male-specific decline in $\mathrm{B}$ cell proportions after age 65 (Wilcoxon $p=1.6 \mathrm{e}^{-05}$ ), resulting in a significant difference in $B$ cell proportions between older men and older women (Wilcoxon $p=0.0032$ ) (Fig. 1d, Supplementary Table 4). We did not detect significant aging- or sex-related changes in $\mathrm{CD}_{14}{ }^{+}$monocytes (Kruskal-Wallis $p$-value $=0.32$ ) $($ Fig. $1 \mathrm{~d})$. These four cell types constitute the majority of PBMCs. However, PBMCs also include NK cells (5\%), DCs (1-2\%), CD16 ${ }^{+}$ monocytes $(1 \%)$, ILCs $(<1 \%)^{15}$. Among these, the abundance of $\mathrm{CD}^{+} 6^{+}$monocytes have been reported to increase with age, whereas ILC and DC subsets and CD56 ${ }^{+}$NK cells decrease with age $^{16}$. Furthermore, $\mathrm{CD}_{6} 9^{+} \mathrm{CD}_{16}{ }^{+} \mathrm{NK}$ cells were more abundant in men, whereas subsets of T cells and ILCs showed more abundance in women.

We studied subsets of $\mathrm{CD}^{+}$and $\mathrm{CD}^{+} \mathrm{T}$ cells in a subgroup of young and older individuals $(n=80)$ : naive $\left(\mathrm{CD}_{4} 5 \mathrm{RA}^{+}\right.$, $\left.\mathrm{CCR}^{+}\right)$, central memory $\left(\mathrm{CD} 4 \mathrm{RA}^{-}, \mathrm{CCR}^{+}\right)$, effector memory $\left(\mathrm{CD}_{4} \mathrm{RA}^{-}, \mathrm{CCR}^{-}\right)$, and effector memory RA (CD45RA ${ }^{+}$, CCR7 $^{-}$). Expectedly, naive $\mathrm{T}$ cell frequencies decreased with age, particularly in $\mathrm{CD}^{+} \mathrm{T}$ cells (Supplementary Fig. 1d, e). As previously reported ${ }^{17}$, women had more naive $\mathrm{T}$ cells compared to men. For example, $\sim 13.2 \%$ of PBMCs were naive CD $4^{+} \mathrm{T}$ cells in young women, compared to $\sim 7.8 \%$ in young men (Wilcoxon $p=0.00057)$ despite the cohort of young women being slightly older (Supplementary Tables 1,4). Women have elevated thymic function compared to men at all ages ${ }^{18}$, potentially explaining observed sex-differences in naive T cells. Sex- and agerelated changes in other $\mathrm{T}$ cell subsets were not as significant and as consistent as the changes in naive $\mathrm{T}$ cells (Supplementary Fig. 1d, e).

Using generalized linear models (GLM), we studied the relationship between age and sex of individuals and the variation in their PBMC compositions, which confirmed that age is most 
a
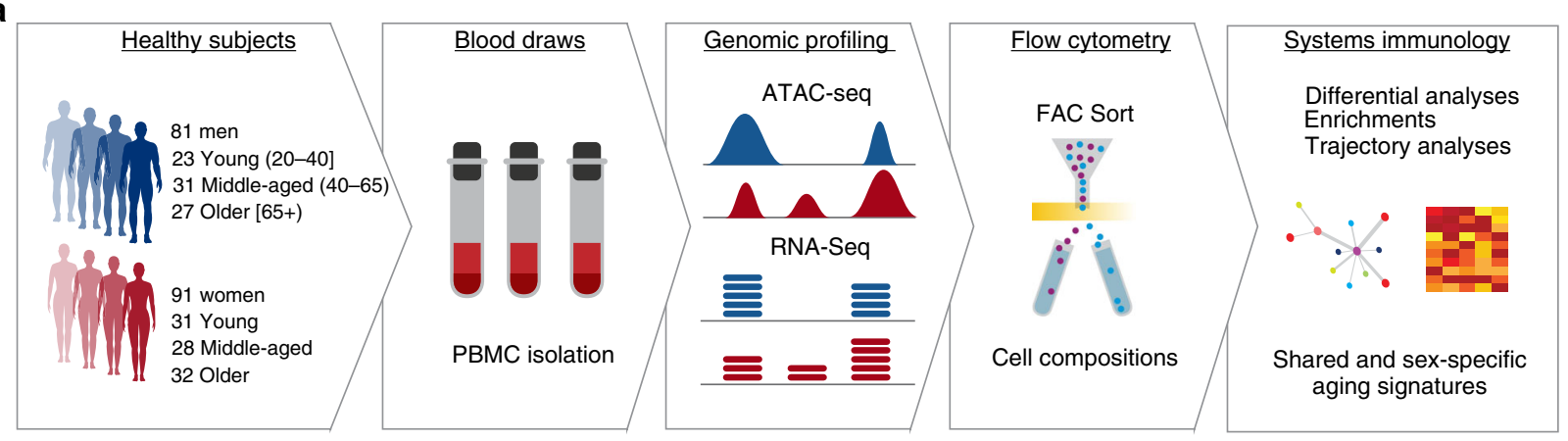

b

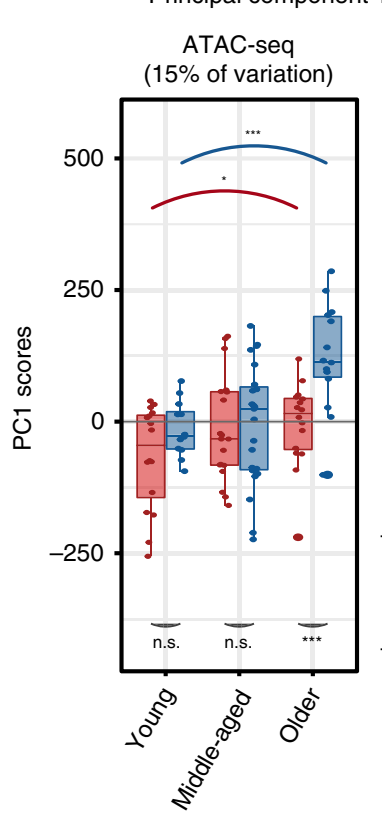

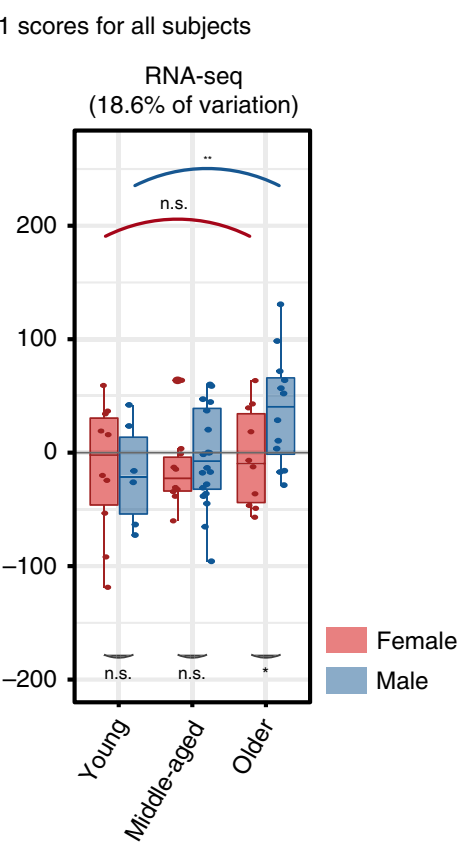

C

Immune module annotation of PC1 (aging) axis

ATAC-seq RNA-seq

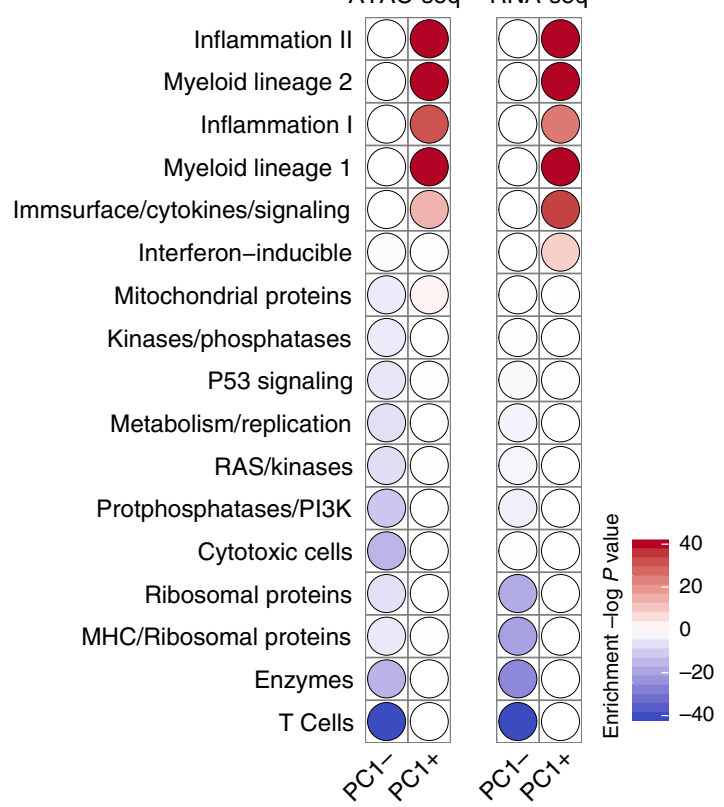

d

Percent of major cell types within PBMCs across age groups $(n=128)$

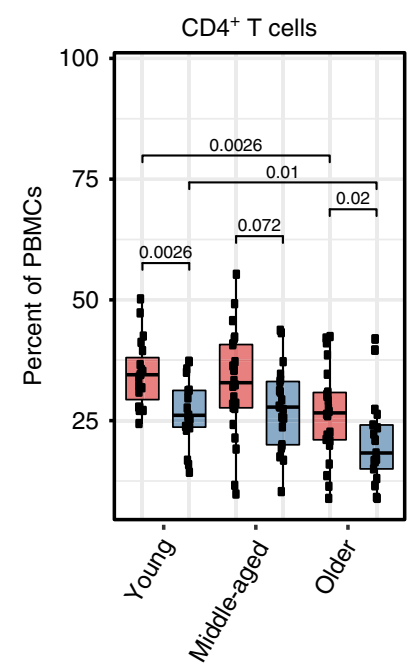

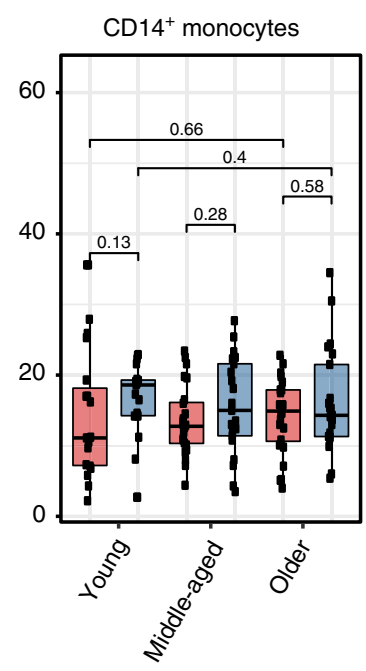

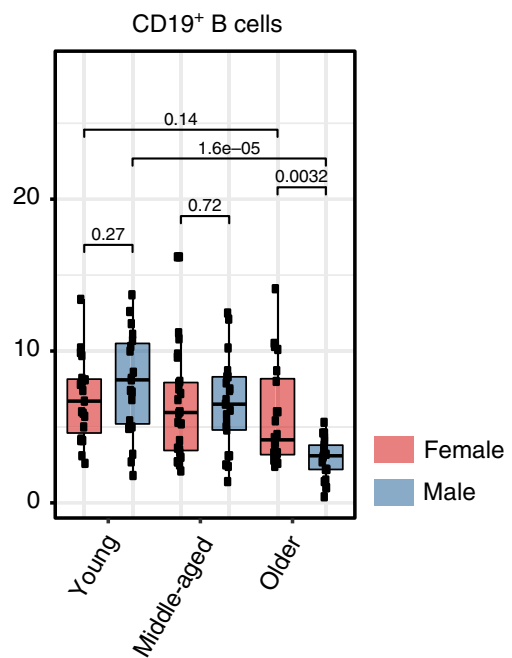

strongly associated with $\mathrm{CD}^{+} \mathrm{T}$ cell proportions, especially for naive cells (Supplementary Fig. 1f). Whereas sex is most strongly associated with naive $\mathrm{CD}^{+} \mathrm{T}$ cells; women have higher proportions compared to men (Supplementary Fig. 1d, f). Finally, we observed that $\mathrm{B}$ cell proportions are associated with joint effects of sex and age (Supplementary Fig. 1f), due to the malespecific decline in B cell proportions after age 65 (Fig. 1d). Together, these data uncovered shared and sex-specific changes in PBMC cell compositions with age, some of which underlie genomic changes. 
Fig. 1 Age is the main driver of variation in PBMC genomic data. a Schematic summary of study design: PBMCs were isolated from blood samples of 172 healthy community-dwelling individuals (ages 22-93), from which ATAC-seq, RNA-seq and flow-cytometry data were generated. b Principal component 1 scores (PC1) were calculated for each individual from ATAC-seq (left) and RNA-seq (right) principal component analyses (PCA) results. Marker genes are selected using PC1 scores that are $\geq 25$ th percentile: top positive scores (ATAC-seq $n=9392$ peaks, RNA-seq $n=1413$ genes) and top negative scores (ATAC-seq $n=10,151$ peaks, RNA-seq $n=1675$ genes). PC1 scores from ATAC-seq and RNA-seq data increased with increasing age. Furthermore, we detected differences in PC1 scores of sexes in older subjects, where older men had higher scores than older women. c Functional enrichment of PC1-related genes based on immune modules ${ }^{22}$. Note that myeloid/inflammation-related genes were associated with high and positive scores, whereas adaptive immunity/lymphoid related genes were associated with high and negative PC1 scores. These enrichments align well with an age-related increase in the myeloid lineage and inflammation, and an age-related decline in naive T cell activity. Hypergeometric test was used to calculate enrichment $p$-values. d Flow cytometry data from 128 individuals that reflect proportions of major cell types within PBMCs. Subjects were stratified based on age group and sex: Young: 22-40, Middle-aged: 41-64, Older: $65+$ years old. Wilcoxon rank-sum test was used to compare data from female $(n=67)$ and male $(n=61)$ subjects. Note that $\mathrm{T}$ cell proportions decline with age in both sexes, whereas the decline in $\mathrm{B}$ cell proportions is specific to older men. Box plots represent median and IQR values, whiskers extend to 1.5 times the IQR; ${ }^{\star \star \star} p<0.001,{ }^{\star \star} p<0.01,{ }^{\star} p<0.05$, n.s.: non-significant. Source data are provided as a Source Data file for (c).

Shared and sex-specific chromatin accessibility signatures of aging. We detected ATAC-seq peaks that are differentially accessible (DA) between young and older individuals ${ }^{19}$ (FDR $5 \%)$. In men, 10,196 peaks were DA with age (13\% of tested: 5782 closing, 4414 opening), compared to 4516 DA peaks in women (5.8\% of tested: 3309 closing, 1207 opening) (Fig. 2a, Supplementary Table 5). Male and female DA peaks significantly overlapped, despite thousands of sex-specific loci associated with aging. Furthermore, a significant correlation was detected between sexes in terms of their epigenomic aging signatures (Pearson $r=0.445, p<0.001$ ). DA peaks corresponded to 6612 and 3634 genes in men and women, respectively (Supplementary Fig. 2b). Functional annotation of these peaks using Roadmap chromatin states in immune cells ${ }^{20}$ revealed that closing peaks were mostly regulatory elements (promoters, enhancers) shared across cells, especially $\mathrm{T}$ cell subsets (Fig. 2b, Supplementary Fig. 2c). In contrast, opening peaks were mostly enhancers of $\mathrm{CD}_{14}{ }^{+}$monocytes and $\mathrm{CD} 56^{+} \mathrm{NK}$ cells in both sexes (Fig. 2b, Supplementary Fig. 2d). As previously reported ${ }^{5}$, there were more 'quiescent' loci (i.e., loci without functional annotation) among opening peaks, which may be due to global chromatin deregulation with age ${ }^{21}$.

To identify immune cells/functions affected by aging, we studied changes at cell-specific loci (i.e., loci active in one cell type) (Supplementary Fig. 2c). In both sexes, opening peaks were associated with monocyte-, NK, and memory $\mathrm{CD}^{+}{ }^{+}$cell-specific loci, whereas closing peaks were associated with naive $\mathrm{T}$ cellspecific loci (Fig. 2c, Supplementary Fig. 2e, f). Despite these similarities, a greater number of cell-specific loci were affected with aging in men. Notably, 960 monocyte-specific peaks significantly gained accessibility with aging in men, whereas only 64 such loci were detected in women. Only B cells showed the opposite changes between sexes, where B cell-specific loci were more likely to be among opening peaks in women and among closing peaks in men (Fig. 2c). Fold change distributions and enrichment analyses confirmed these results. Interestingly, closing peaks were enriched in B cell-specific loci only in men (Supplementary Fig. 2e, f). Immune module ${ }^{13,22}$ and WikiPathways enrichment analyses uncovered that inflammation-related modules/pathways were associated to chromatin opening in both sexes (Supplementary Table 6) (e.g., MyD88 cascade (WP2801) pathway). In contrast, closing peaks were significantly associated to modules/pathways related to T cells, notably the IL-7 Signaling pathway as we previously observed ${ }^{5}$. Several molecules in this pathway were associated to chromatin closing in both sexes including IL7R, JAK1, STAT5B, and PTK2B (Supplementary Table 6, Supplementary Fig. 3 for more examples). Together, these data uncovered an epigenomic signature of aging shared between sexes, which include gains in chromatin accessibility for pro-inflammatory processes, monocytes and cytotoxic cells (NK, $\mathrm{CD}^{+}$memory) and losses in accessibility for naive $\mathrm{T}$ cells. Interestingly, these changes were more pronounced in men, despite cohorts being comparable for age, frailty, and BMI (Supplementary Fig. 1g, Supplementary Table 1). Furthermore, we discovered that $\mathrm{B}$ cells age differently between sexes, where a significant loss in chromatin accessibility was detected only in men.

Correlated aging-related changes in transcriptomes and epigenomes. From PBMC RNA-seq data, we identified 918 differentially expressed (DE) genes in women (539 up, 379 down) and 791 genes in men (510 up, 281 down) (FDR 10\%) ${ }^{19}$ (Supplementary Fig. 4a, Supplementary Table 7). DE genes overlapped significantly between sexes. For example, 201 downregulated genes were shared (Chi-square $p$-value $=8.12 \mathrm{e}-149$ ) and included important $\mathrm{T}$ cell molecules (e.g., TCF7, LEF1, CCR7) (Supplementary Fig. 4b). Annotations using cell-specific marker genes from PBMC scRNA-seq data and sorted cell transcriptomes ${ }^{10}$, revealed that upregulated genes were most significantly enriched in marker genes for NK (Fig. 2e), monocytes and activated CD8 ${ }^{+}$ $\mathrm{T}$ cells (Supplementary Fig. 4c, d, Supplementary Table 8). In contrast, downregulated genes were most significantly enriched in naive $\mathrm{T}$ cell marker genes (e.g., CD27, CCR7, LEF1) in both sexes (Fig. 2e, f, Supplementary Table 8). Similar to the ATAC-seq data (Fig. 2c), aging-related gene expression changes in the $\mathrm{B}$ cell compartment were sex dimorphic. B cell-specific genes, especially naive $\mathrm{B}$ cells (e.g., $B C L 7 A, P A X 5, C D 79 A$ ) were downregulated with age only in men (Fig. 2e, Supplementary Fig. 4d, e), potentially due to changes in cell composition (Fig. 1d). Notably, aging-related transcriptomic and epigenomic changes correlated significantly in men (Pearson $r=0.31, p<10 \mathrm{e}^{-4}$ ) and in women (Pearson $r=0.21, p<10 \mathrm{e}^{-4}$ ). Enrichment analyses confirmed the upregulation of genes associated to cytotoxic cells and inflammation in both sexes (e.g., GZMB, PRF1, NKG7 in women) (Supplementary Fig. 4f) and downregulation of T cell genes (e.g., LEF1, TCF7, CCR7 in both sexes) (Supplementary Table 8). These results demonstrate that age-related changes in epigenomes and transcriptomes correlated significantly and uncovered an agerelated shift in PBMCs from adaptive to innate immunity in both sexes, albeit more pronounced in men.

Age-related changes in monocyte- and B cell-associated loci differ between sexes. Age-related changes in ATAC-seq (Fig. 3a, Pearson $r=0.435, p<0.001$ ) and RNA-seq data (Fig. 3b, Pearson $r=0.484, p<0.001)$ positively correlated between sexes. However, enrichment analyses using Roadmap ${ }^{20}$ and scRNA-seq data revealed that similarity between sexes depends on the cell type 
a

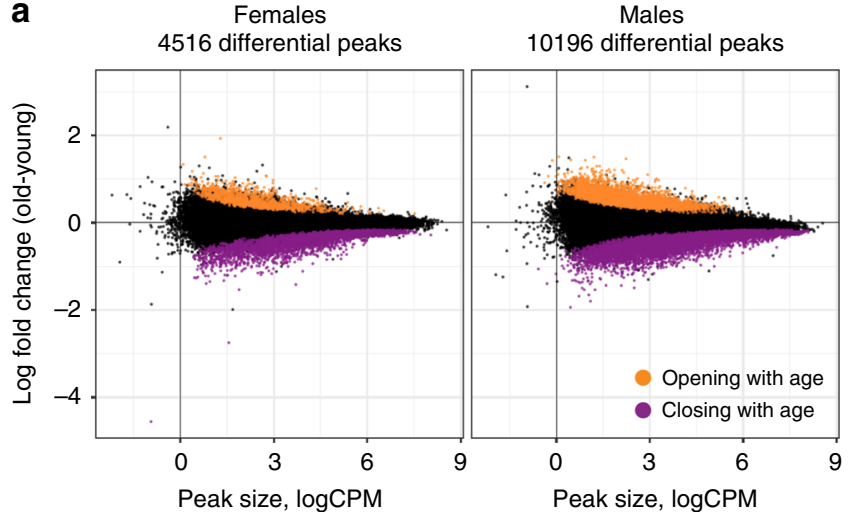

C

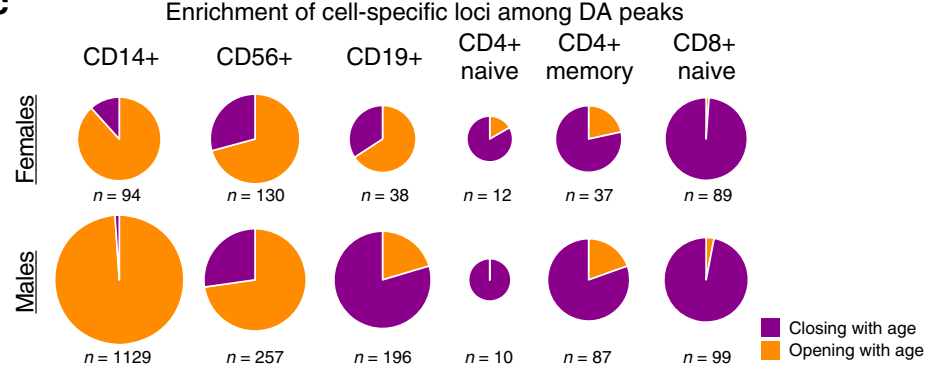

b

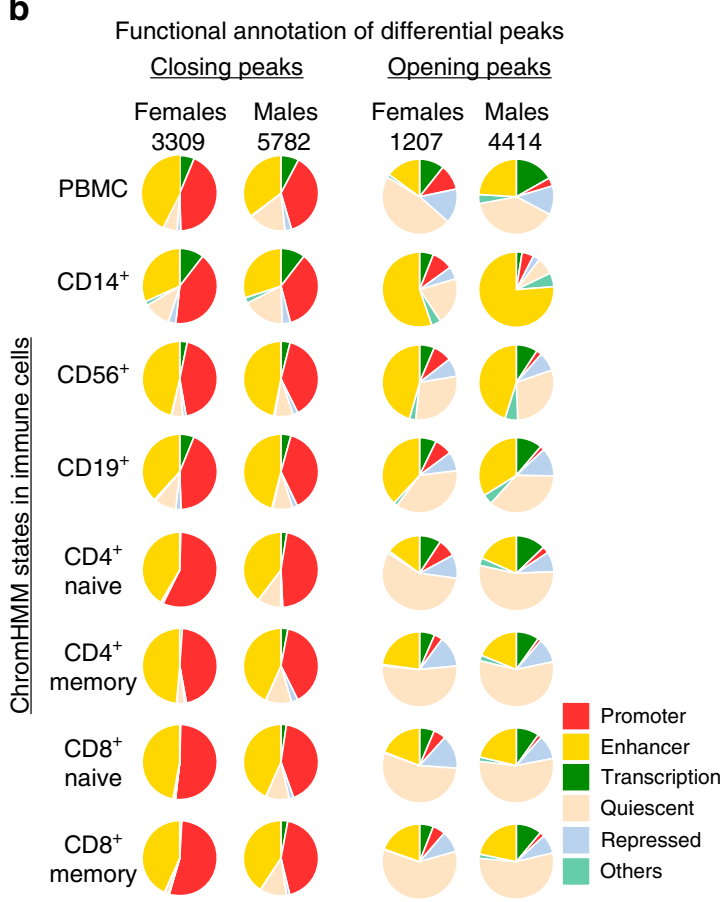

d

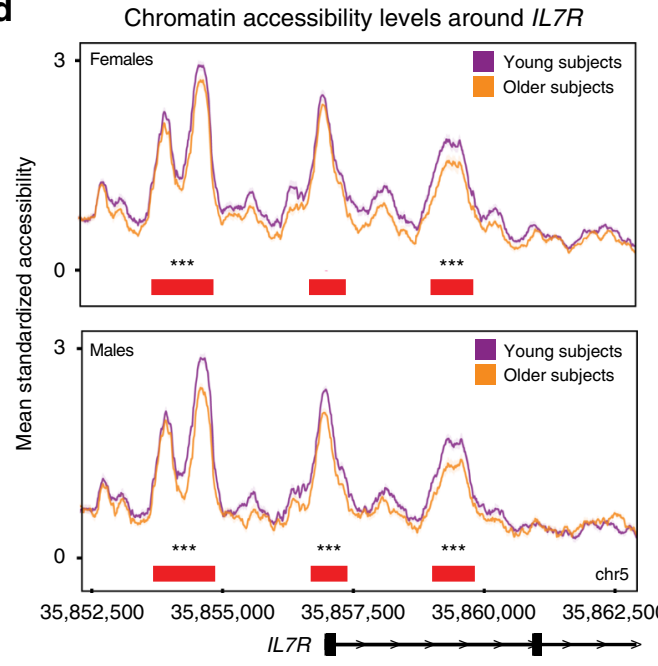

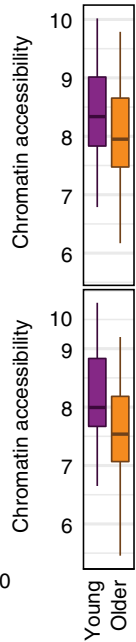

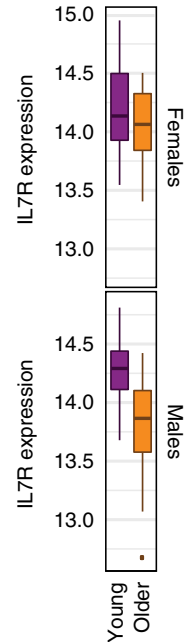

e

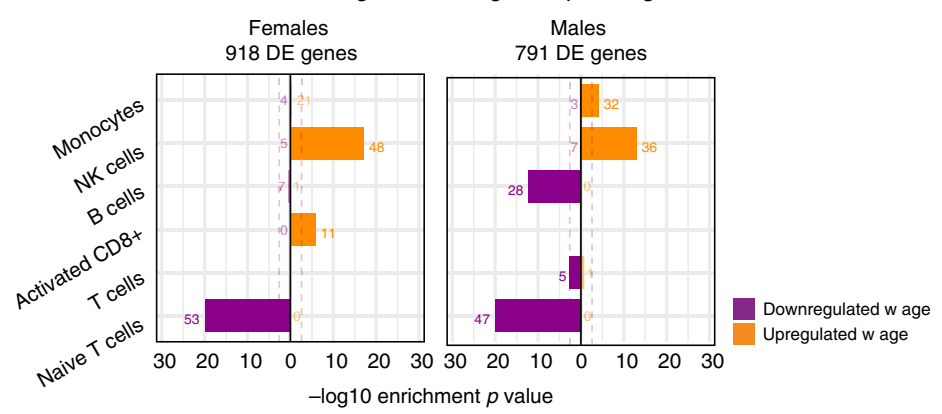

f

Expression of $\mathrm{T}$ cell associated genes Females Males
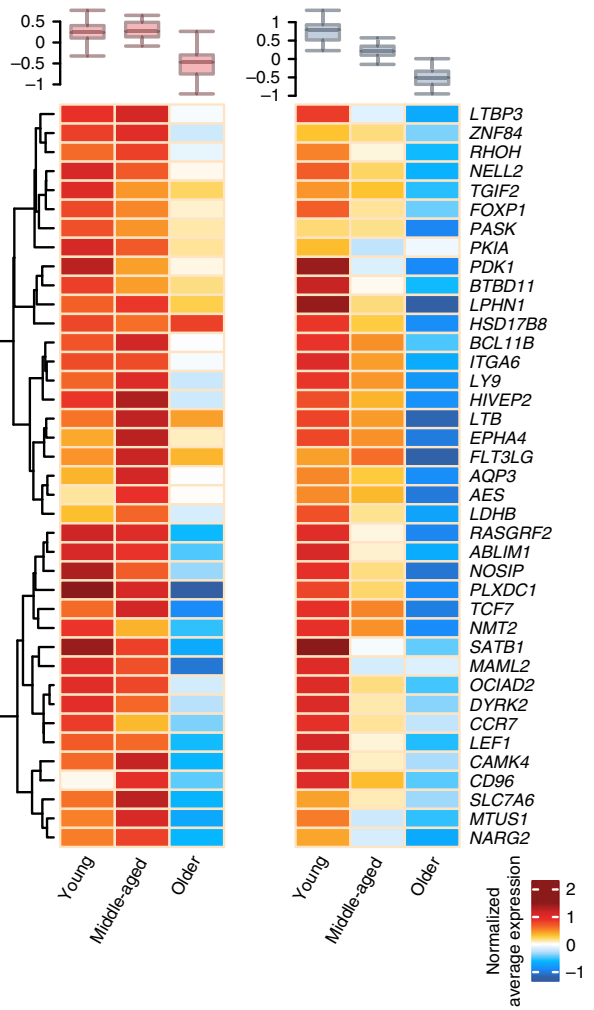

(Fig. 3c, d). For example, decreases associated with naive $\mathrm{T}$ cells were highly correlated between sexes both in transcriptomic (Pearson $r=0.698$, Fig. 3d) and epigenomic data (Pearson $r=$ 0.705 , Fig. 3c, Supplementary Fig. 5a), likely due to the agerelated loss of naive $\mathrm{T}$ cells (Supplementary Fig. 1d, e). These included shared changes observed in $I L 7 R$ - a receptor associated to aging (Fig. 2d $)^{5}$-chemokine receptor CCR7, co-receptor CD8A, and TFs TCF7 and LEF1 (Supplementary Table 6). Gene expression levels of these molecules also decreased with age in both sexes (Figs. 2d, 3e). Similarly, changes in cytotoxic cells were highly correlated between sexes (Pearson coefficient NK cells: RNA-seq $r=0.621$, ATAC-seq $r=0.521$; cytotoxicity module: 
Fig. 2 Shared and sex-specific epigenomic signatures of aging. a Fold change (older-young) vs. read counts per million (logCPM) from ATAC-seq differential analyses in women (left) and men (right) based on 78,167 ATAC-seq peaks (5\% FDR after Benjamini-Hochberg P-value adjustment, 58 subjects). Differentially opening/closing peaks are represented in orange/purple. b Functional annotations of differentially accessible (DA) peaks in men and women using chromHMM states in PBMCs and cell subsets. Closing peaks tend to be enhancers/promoters across all cell types, whereas opening peaks tend to be enhancers in monocytes and NK cells. c Distribution of DA peaks among cell-specific loci from chromHMM states. Innate cellspecific loci (monocytes, NK cells) are enriched in opening peaks, T cell-specific loci are enriched in closing peaks. B cell-specific loci are more likely to be opening peaks in women and closing peaks in men. $\mathbf{d}$ Left: Average chromatin accessibility profiles (grouped by age and sex) around the IL7R locus-is associated with chromatin closing with age in women (top, $n=32$ ) and men (bottom, $n=26$ ). Red bars indicate peaks and their significance value from differential analyses $\left({ }^{\star} p<0.05,{ }^{\star *} p<0.01,{ }^{\star \star *} p<0.001\right.$, no symbols: non-significant). Right: Normalized chromatin accessibility (combined from three peaks shown in the figure) and gene expression levels for IL7R in young $(n=16)$ and older $(n=16)$ women (top) and young ( $n=12)$ and older ( $n=14)$ men (bottom). In both sexes, chromatin accessibility and gene expression levels decline with age albeit more significantly in men. e Enrichment of differentially expressed (DE) genes using cell-specific gene sets from scRNA-seq data. Enrichment $p$-values are calculated using hypergeometric test. Gene expression programs for innate cells (NK, monocytes) are activated, whereas programs for $\mathrm{T}$ cells are inactivated in both sexes. B-cell specific genes were downregulated specifically in men. $\mathbf{f}$ Average expression levels of T cell-specific genes grouped by age group and sex. Note the decline in both sexes. Box plots on the top summarize the data from all genes represented in the heatmap. All box plots represent median and IQR (interquartile range) values, with whiskers extending to 1.5 times the IQR. Source data are provided for $(\mathbf{a}, \mathbf{b}, \mathbf{c}, \mathbf{e})$.

RNA-seq $r=0.772$, ATAC-seq $r=0.675$ ) (Fig. 3c, d, Supplementary Fig. 5b, c) and their activity increased with age in both sexes. These included granzyme B (GZMB) and perforin (PRF1), which have complementary cytotoxic functions, and KLRF1, which is selectively expressed in NK cells ${ }^{23}$ relative to cytotoxic $\mathrm{T}$ cells (CTLs) (Fig. 3e). Combined with our previous study, these results suggest that both CTLs and NK cells contribute to the increased cytotoxicity with age in both sexes. In contrast, agerelated changes associated to monocytes and $\mathrm{B}$ cells showed the lowest correlation between sexes (Fig. 3c, d, Supplementary Fig. 5b, c). In monocytes, a majority of peaks/genes were activated with age in both sexes; however, the magnitude of activation was more pronounced in men (Fig. 2c), which reduced the correlations (Pearson coefficient RNA-seq $r=0.267$, ATAC-seq $r=$ 0.171 ) (Fig. 3c, d). These included pro-inflammatory cytokines IL18, ILIB and S100A8/S100A9 genes that modulate inflammatory responses and serve as potential biomarkers of inflammation-related diseases ${ }^{24}$. In $\mathrm{B}$ cells, the distinction between sexes stemmed from the male-specific downregulation/ chromatin closing of B-cell specific loci/genes (Pearson coefficient RNA-seq $r=0.195$, ATAC-seq $r=0.017$ ) (Fig. 3c, d), including the chemokine receptor CXCR4 and $\mathrm{B}$ cell receptor CD79A (Fig. 3e, Supplementary Table 6).

These data revealed that the similarity of the aging signatures between sexes depends on the cell type. Age-related changes in naive $\mathrm{T}$ cells (loss) and $\mathrm{NK}$ and memory $\mathrm{CD}^{+} \mathrm{T}$ cells (gain) were similar. However, gains in monocyte-specific loci were greater in men, which contributed to the sex-differences. B cell-specific loci lost accessibility/expression with age only in men, representing the most sex-dimorphic aging pattern.

Chromatin accessibility and gene expression changes over adult lifespan. We uncovered chronological trends in epigenomic/transcriptomics maps using Autoregressive Integrated Moving Average (ARIMA) ${ }^{25}$ models by first finding the bestfitting ARIMA model to each peak/gene as a function of age and selecting the ones that displayed a chronological trend significantly different than random fluctuations. This uncovered 13,297 and 13,295 ATAC-seq peaks with a temporal trend in women and men, respectively, grouped into three major clusters (Fig. 4a, Supplementary Table 9). Cluster1 (F1/M1) included peaks losing accessibility with age, whereas cluster2 and cluster3 included peaks gaining accessibility with age (F2/M2 and F3/M3). In cluster3, the gains occurred earlier $(\sim 10-20$ years $)$ than in cluster2.

Enrichment analyses revealed that, in both sexes, clusterl was associated to $\mathrm{T}$ cell-related loci, particularly naive $\mathrm{T}$ cells (Fig. $4 \mathrm{~b}$,
Supplementary Fig. 6a). Interestingly, cluster1 was significantly enriched in B cell-specific regions only in men, reinforcing a male-specific decline in B cells. Cluster2 was most significantly enriched in monocyte-specific regions in both sexes, albeit more significantly in men. Finally, cluster 3 was most significantly enriched in NK cell-specific regions in both sexes, more significantly in men. Although less significant, we also detected female-specific enrichment of B cell-specific regions in cluster2 and cluster3, contrasting the declines in men (Figs. 2c, 4b).

ARIMA models revealed temporal transcriptomic patterns consistent with the epigenomic patterns. 1,068 and 1,471 genes had temporal trends across human lifespan respectively in women and men (Fig. 4c, Supplementary Table 9). We grouped these genes into three clusters (men: M1-3, women F1-3) based on the similarity of trends. Enrichment analyses ${ }^{10}$ revealed changes shared between sexes including downregulation of naive $\mathrm{T}$ cell genes and upregulation of monocyte, NK, and effector T cell genes. Similar to the epigenomic trends, temporal genes of men and women differed for the $\mathrm{B}$ cell compartment, due to a male-specific downregulation of $B$ and plasma cell-specific genes (Fig. 4d, Supplementary Fig. 6b) and a female-specific upregulation -albeit weaker- of plasma B cell-specific genes. These data suggest that, other than for B cells, temporal epigenomic/ transcriptomic trends are shared between sexes and composed of genomic declines in $\mathrm{T}$ cell-associated loci and increases in monocytes and NK cell-associated loci.

PBMCs go through rapid epigenomic changes at two discrete periods during adult lifespan. To study whether temporal epigenomic changes are acquired gradually over lifespan or more rapidly at certain ages, we detected age brackets during which abrupt changes take place, referred to as breakpoints. Within each temporal cluster (Fig. 4a), we compared epigenomic profiles observed at ages immediately preceding and succeeding a given age (e.g., 5 years before/after) and calculated a $p$-value for differences between the windows. By using a moving window approach with varying window sizes, we ensured robustness to outliers, sample sparsity at certain ages, and temporal fluctuations (Supplementary Fig. 6c). Finally, for each age, we calculated a combined $p$-value from different windows, where a smaller $p$-value indicates rapid epigenomic changes at that age. Due to RNA-seq sample sparsity, these analyses were restricted to ATAC-seq samples.

These analyses revealed two periods in adult lifespan during which rapid changes occur: (i) a timepoint in late-thirties/earlyforties, and (ii) a later timepoint after 65 years of age (Fig. 4e, Supplementary Fig. 6c). The earlier breakpoint was detected 
a

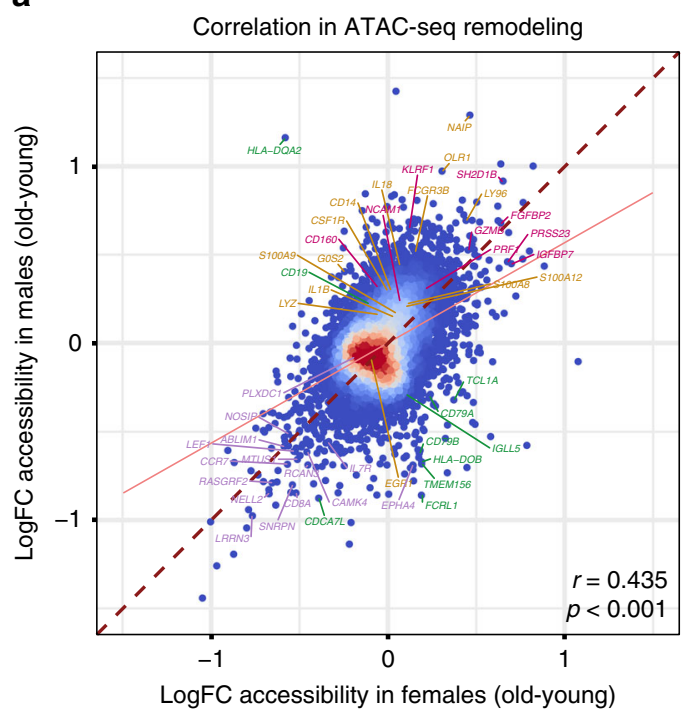

b

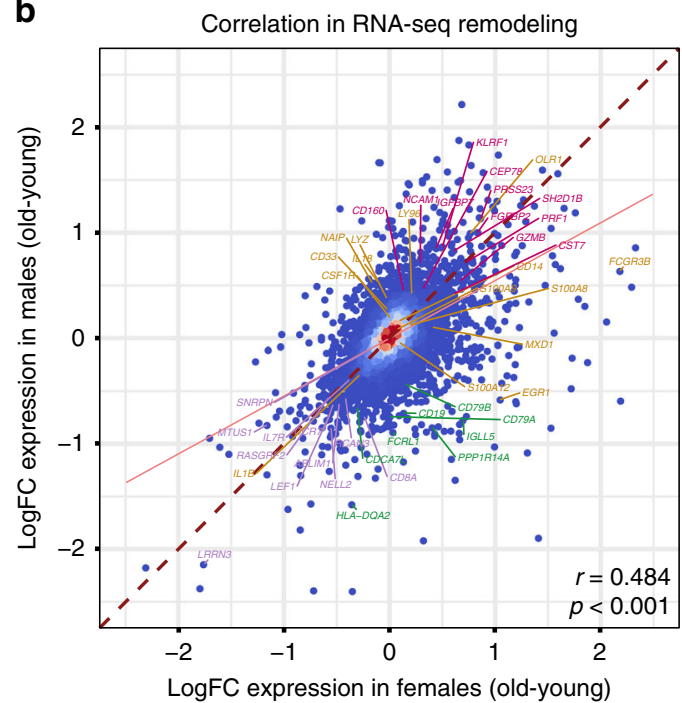

C

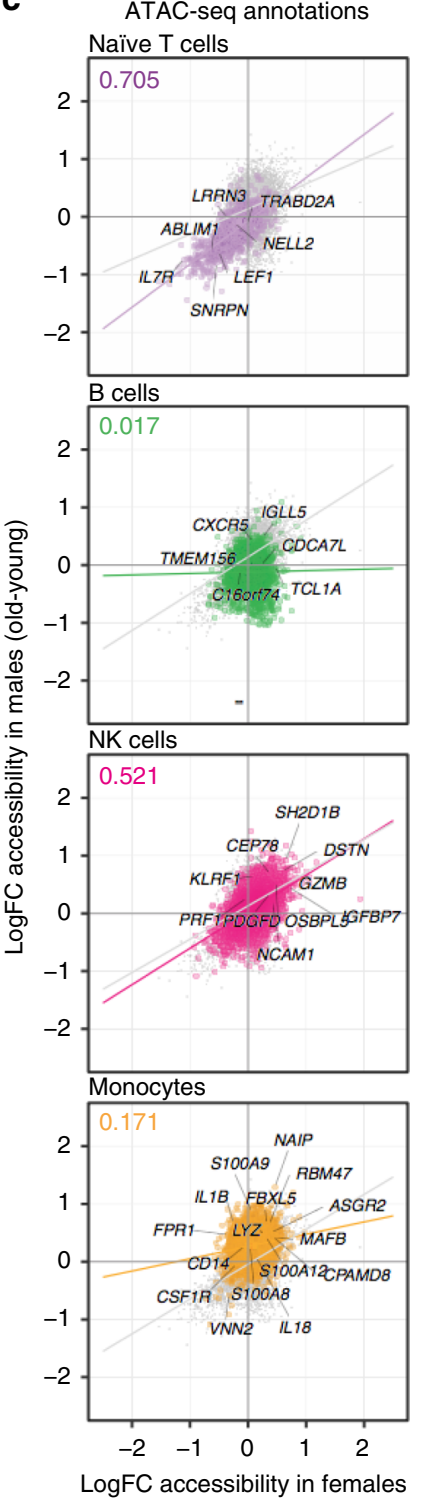

d

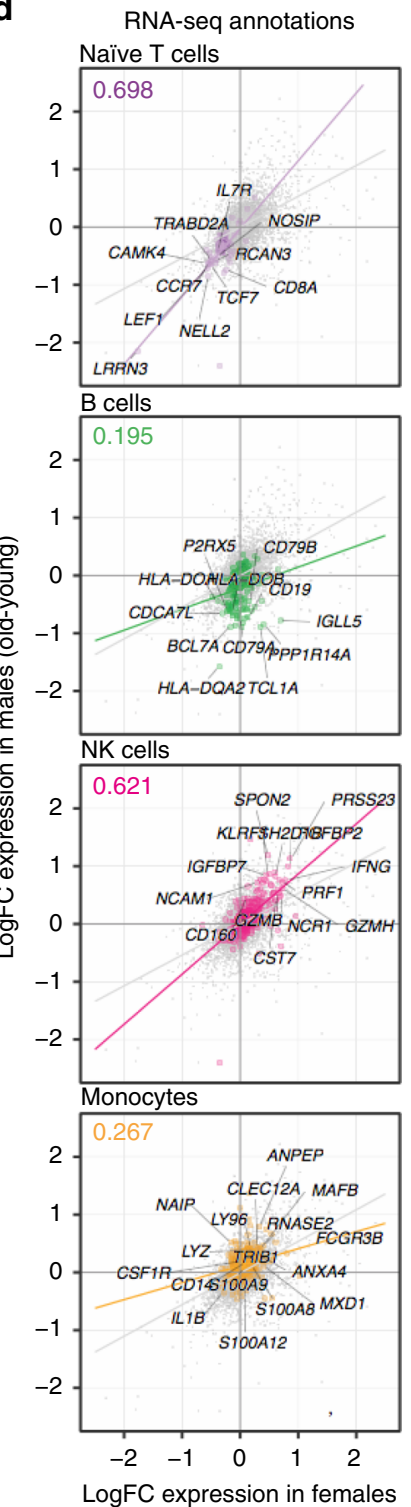

e
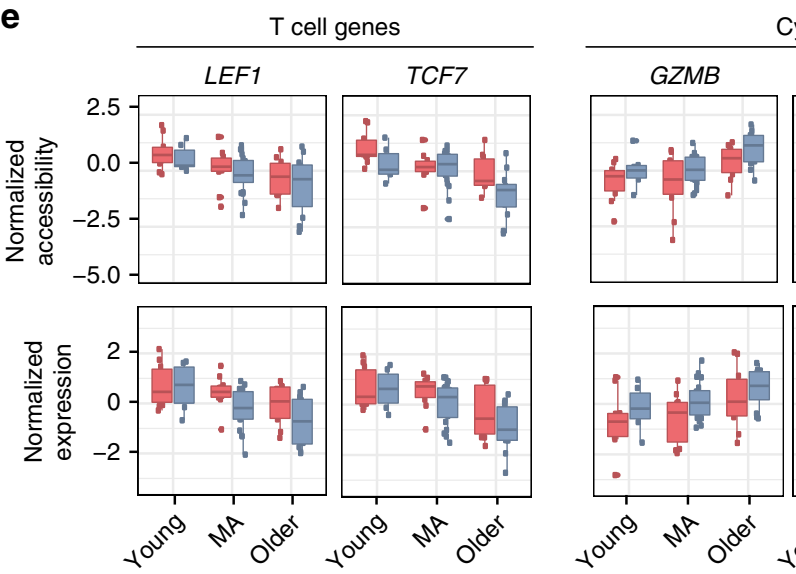

Cytotoxic cell genes
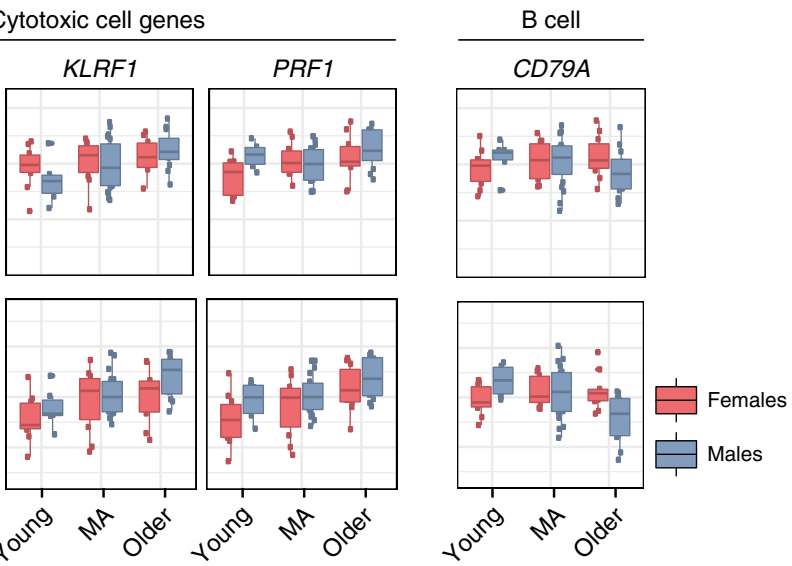

between ages 38-41 in women across three clusters and ages 40-42 in men in cluster1 and cluster3. Magnitude (breakpoint $p$-values, Fig. $4 \mathrm{e}$ ) and timing of changes for this breakpoint were similar between sexes. Together with Fig. 4a, this timepoint matched the start of aging-related epigenomic changes, i.e., the start of chromatin accessibility losses in cluster1 and gains in

cluster2/3. The later breakpoint was detected between ages 66-71 in women across three clusters (Fig. 4e, Supplementary Fig. 6c). Interestingly, in men, this breakpoint was observed earlier (ages 62-64). The magnitude of changes for the second breakpoint was more pronounced in men than in women. Combined with Fig. 4a, this breakpoint coincided with the acceleration of epigenomic 
Fig. 3 Sex-dimorphic changes in monocyte- and B cell-associated loci. Correlation of age-related ATAC-seq (a) and RNA-seq (b) remodeling between women and men PBMCs. Note the overall large and positive Pearson correlation coefficients. Genes are associated to ATAC-seq peaks based on nearest TSS, and are color coded in both plots according to their association to immune modules (purple: T cells, green: B cells, pink: NK cells, yellow: monocytes). Only regulatory (TSS/enhancer) peaks are included and peaks-genes are matched between both plots $(n=10,707$ loci). Blue-red gradient on data points represents their relative local density. (c) Correlation between sexes for age-related ATAC-seq remodeling stratified by cell-specific loci from chromHMM annotations. Note that the highest correlation is observed in naive T cells ( $n=833$ peaks), which is associated with negative fold changes (i.e., loss in chromatin accessibility with age) in both sexes. Chromatin remodeling correlates the least between sexes for B cell- ( $n=1645$ peaks) and monocytespecific loci ( $n=6861$ peaks), with NK cells ( $n=3,008$ peaks) showing a positive trend in both sexes. $\mathbf{d}$ Correlation between sexes for age-related RNAseq remodeling stratified by cell-specific genes from single-cell RNA-seq data. Note that the highest correlation is observed in naive T cells ( $n=70$ genes), which is associated with negative fold changes (i.e., downregulation with age) in both sexes. On the other hand, NK cells ( $n=403$ genes) are highly correlated between sexes and associated with increased expression with age in both sexes. In agreement with ATAC-seq data, gene expression remodeling correlates the least between sexes for B cell- $(n=144)$ and monocyte-specific genes $(n=748)$. Values at the top left corner represent the Pearson correlation coefficient between sexes only for the genes/loci associated to that cell type. e Normalized expression and accessibility levels for important molecules associated to T, cytotoxic, and B cells. LogFC = mean log2 of fold changes between old and young samples; MA = middle-aged. Box plots represent median and IQR values, with whiskers extending to 1.5 times the IQR. Source data are provided as a Source Data file for (a-d).

changes, such as a faster loss of chromatin accessibility in cluster1. In summary, breakpoint analyses revealed that although aging-related changes accumulate gradually, there are at least two periods in the adult lifespan during which the immune system undergoes more abrupt epigenomic changes. The first breakpoint manifests itself in both sexes similarly, whereas the second latter breakpoint affects men more strongly and earlier than women. Timing of breakpoints were comparable across clusters, suggesting that cellular interactions might modulate these temporal trends.

Shared temporal genomic patterns between men and women. We detected 3,197 peaks and 180 genes with shared temporal trends between sexes, which grouped into three clusters (Fig. 5a, Supplementary Fig.7a). Cluster1 captured a downward trend in both datasets and was associated with naive $\mathrm{T}$ cells (Fig. 5b, Supplementary Fig. 7b). Similarly, cluster3 exhibited an upward trend and was associated to monocytes and NK cells. Interestingly, cluster2 exhibited opposite temporal patterns between sexes: a downward trend in men and an upward trend in women and was associated to B cells (Fig. 5b, c, Supplementary Fig. 7b).

Further inspection of clusters identified temporal changes for important molecules. Cluster1 included T cell development and signaling genes, e.g., co-stimulatory molecule CD27 and TCF7-a key $\mathrm{TF}$ in $\mathrm{T}$ cell development (Supplementary Table 9). Expression of both molecules decreased with age, the decrease was faster in men (Supplementary Fig. 7d). In contrast, cluster2 exhibited contrasting temporal patterns between sexes and was associated to B and plasma cells, in both ATAC-seq and RNA-seq (Fig. 5b, Supplementary Fig. 7b). These included the E-protein TCF4 (E2.2) that control various aspects of B cell development ${ }^{26}$ and ORAI2 in the B cell receptor-signaling pathway; expression levels of both genes increased in women and decreased in men. Changes in cluster3 included upregulation of JAG2 in both sexes, which is expressed specifically in $\mathrm{NK}$ cells ${ }^{10}$ and plays critical roles in DC-mediated NK cell cytotoxicity ${ }^{27}$ (Supplementary Fig. 7d).

To uncover potential regulators of epigenomic changes, we identified TF motifs enriched in temporal peaks using 1,388 motifs for 681 TFs grouped into 278 families. Fifty of these families were significantly enriched across three clusters (Fig. 5a). These included families that were enriched across all clusters (e.g., a bHLH family motifs for TCF3, TCF4, TAL1) and enriched in a single cluster (Fig. 5d, Supplementary Table 10, see Supplementary Fig. 7e for sex-specific analyses). TFs associated to naive $\mathrm{T}$ cells ${ }^{10}$ were enriched in cluster1 (e.g., NPAS2, TCF7, SOX12, FOXO1). Among these, TCF7 is a potential 'pioneer factor' and can impact the chromatin accessibility levels ${ }^{28}$. Expression levels of these TFs also decreased with age (Fig. 5e). Cluster3 was enriched for motifs for MAFB, CEBPA, CREB5, and NFE2, which are highly expressed in monocytes ${ }^{10}$ and were upregulated with age in PBMCs (Fig. 5f). TF enrichments for cluster2 were not as significant due to the size of this cluster. Combined analyses of temporal trends in men/women uncovered shared changes: (i) downward genomic trends in naive $\mathrm{T}$ cell genes/TFs, including the pioneer factor TCF7; (ii) upward trends for genes/TFs active in monocytes and cytotoxic cells. These analyses further highlighted the stark differences between sexes regarding aging of the B cell compartment.

PBMCs of men and women diverge with age. We compared ATAC-seq and RNA-seq maps of men and women to describe sex-bias in human PBMCs at different age groups. In young and middle-aged subjects, very few differences were detected between sexes. Interestingly, differences between sexes increased after the age of 65 , where 1011 differential peaks and 548 differential genes were detected (Fig. 6a, b). Functional annotations of sexdifferences were conserved between the two assays, revealing a bias towards myeloid lineage particularly for monocytes in older men and a bias towards adaptive cells (B, T) in older women (Fig. 6c, Supplementary Fig. 8a, Supplementary Table 11). Epigenomic enrichments ${ }^{20}$ (Supplementary Fig. 8b, c) confirmed that male-biased peaks were enriched in monocyte-specific loci and female-biased peaks were enriched in $\mathrm{B} / \mathrm{T}$ cell-specific loci, including important signaling molecules (JAK3, STAT5B) (Supplementary Table 11). Furthermore, IL-7 signaling and other B/T cell signaling pathways were more active in PBMCs of older women compared to older men (Supplementary Fig. 6d, Supplementary Tables 12,13). Given that this pathway is downregulated with age in both sexes (Fig. 6d, right table), the difference between older subjects suggest that the age-related decline in T cells is greater in men. In contrast, older men had higher activity for inflammation-related modules compared to older women, including the pro-inflammatory cytokine IL18. Chromatin accessibility levels for inflammation-related genes/loci increases with age in both sexes (Fig. 6d, right table), suggesting that although inflammation increases with age in both sexes, the magnitude is greater in men. In agreement, serum protein measurements from the 500 Human Functional Genomics consortium $^{11}(n=267)$ revealed that older men have higher levels of pro-inflammatory proteins (IL18, IL6) and their receptor antagonists (IL18BP, IL1RA) compared to older women. IL18BP and IL6 levels increased with age in both sexes, however, the increase was greater in men leading to differences between older adults. In contrast, serum IL1RA levels increased specifically in 
a

Temporal peaks in women and men
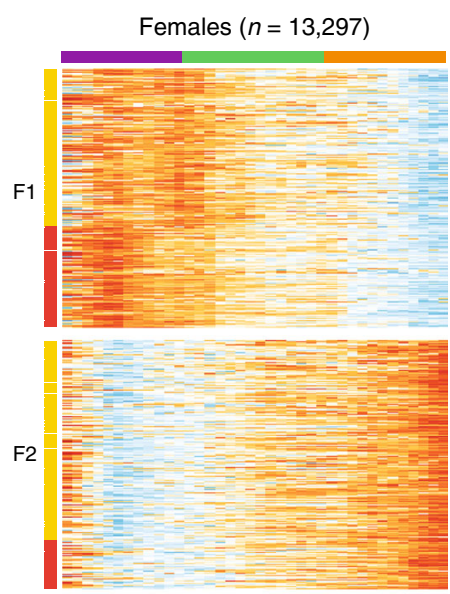

F3

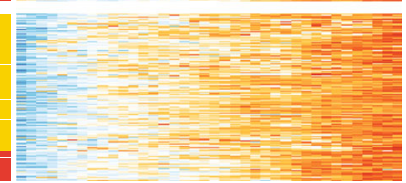

N $\tilde{\omega}$
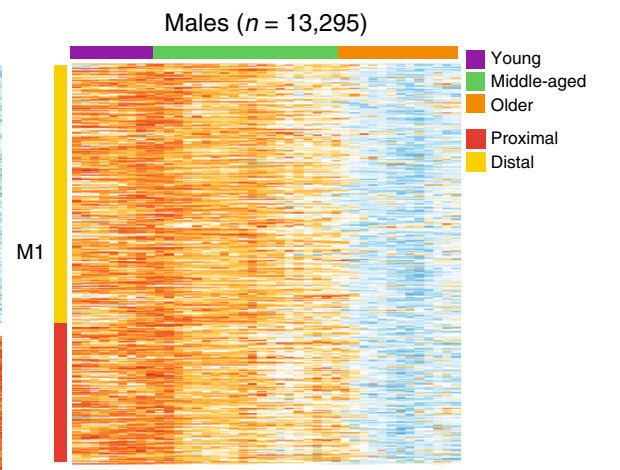

M2

\section{(1)}

M3

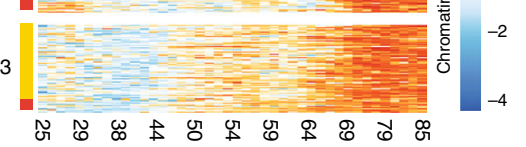

C Temporal transcripts in women and men

Females $(n=1068)$

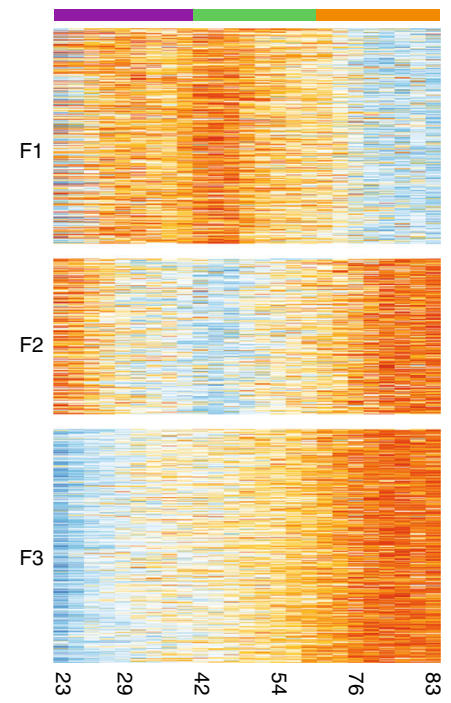

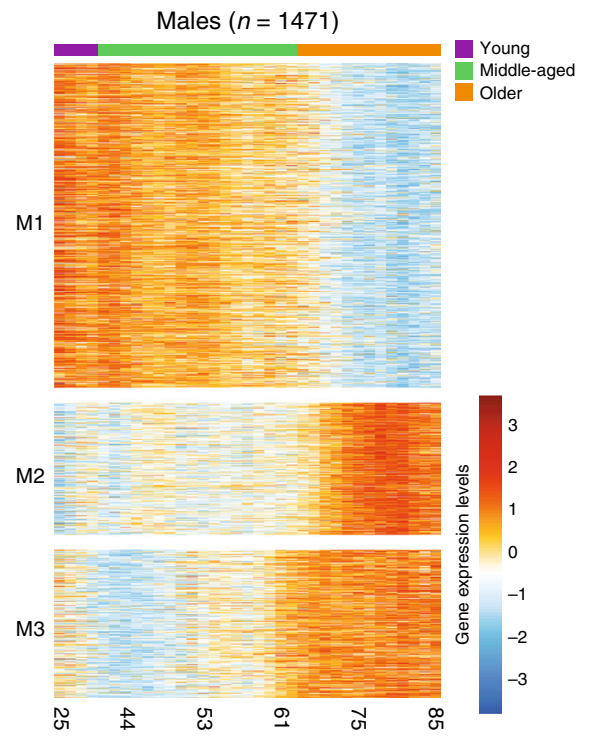

b

Annotation of temporal peaks

ChromHMM annotations

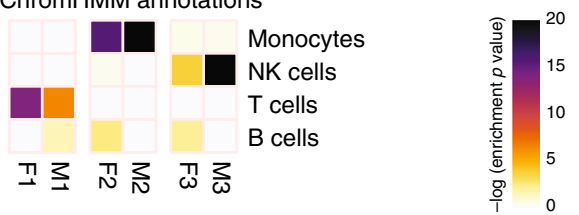

d

Annotation of temporal genes

scRNA-seq annotations

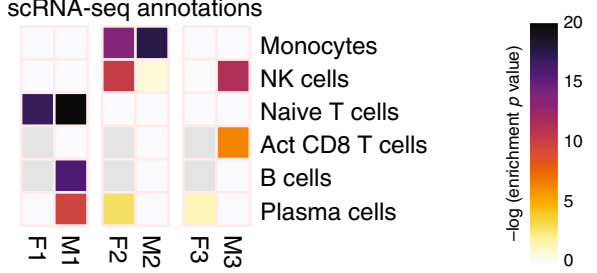

e Breakpoint analyses
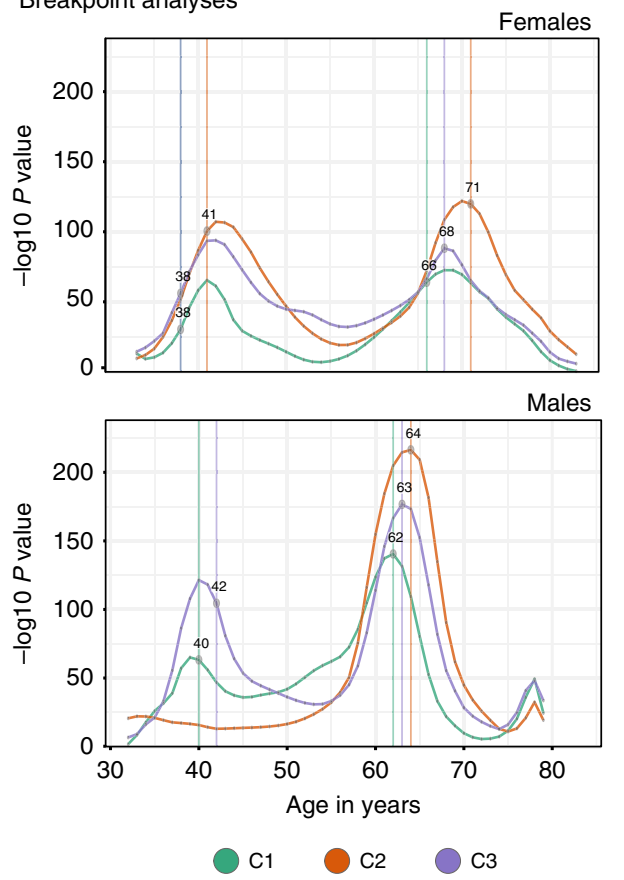

Fig. 4 Epigenome and transcriptome changes over human adult lifespan. a Heatmap of ATAC-seq data (fitted values from ARIMA models) with a significant chronological trend in women (left, $n=13,297$ ) and men (right, $n=13,295$ ), as a function of age in years. Values represent $z$-score normalized accessibility values relative to the row (i.e., peak) mean. K-means clustering was used to group these peaks into three clusters in men and women (M1/F1, M2/F2, M3/F3). Color bar on the top represents discrete age groupings as defined in this study (young, middle-aged, older). Rows are annotated according to their position relative to the nearest TSS: proximal if $<1 \mathrm{kbp}$ distance, distal otherwise. $\mathbf{b}$ ChromHMM state annotations of temporal peak clusters in women (F1-F3) and men (M1-M3). Colors represent hypergeometric enrichment test p-values; light gray cells indicate insufficient number of genes to run an enrichment test. c Heatmap of ARIMA-fitted expression values for genes with a significant chronological trend in women (left, $n=1068$ ) and men (right, $n=1471$ ), as a function of age in years. Three clusters per sex are identified using k-means clustering: F1-3 and M1-3. Values represent $z-$ score normalized expression values relative to the row (i.e., gene) mean. d Annotation of temporal gene clusters using cell-specific gene sets derived from single-cell RNA-seq data. Colors represent hypergeometric enrichment test $p$-values; light gray cells in plot indicate that there were insufficient genes in the gene sets to run an enrichment test. e Inverse log $p$-value distributions from breakpoint analysis for each cluster for women (top) and men (bottom), where curve height indicates magnitude of differences between preceding and succeeding age windows. Note that there are two age brackets where epigenomic changes take place abruptly both in men and women. Points and vertical lines mark median age estimates for a breakpoint integrated over multiple scales, and C1-C3 correspond to temporal clusters M1-M3 or F1-F3 from each sex (see the "Methods" section and Fig. S5c for details). Source data are provided as a Source Data file for $(\mathbf{a}-\mathbf{e})$. 
a Shared temporal peaks $(n=3197)$

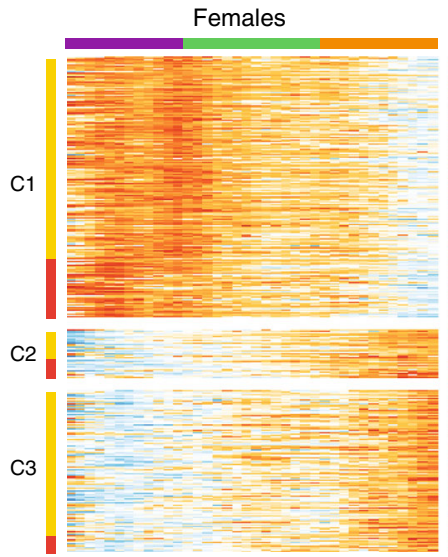

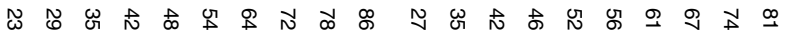

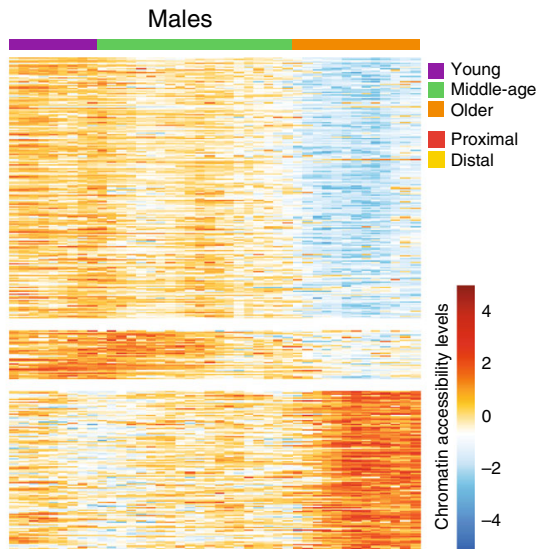

b Annotation of shared peaks ChromHMM annotations

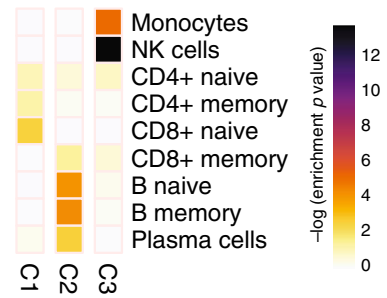

C

Annotation of shared genes

DICE cell-specific annotations

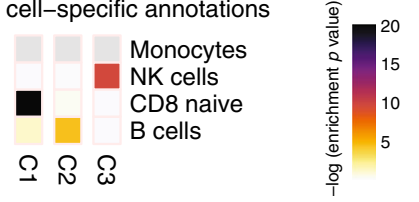

d TF motif family enrichments

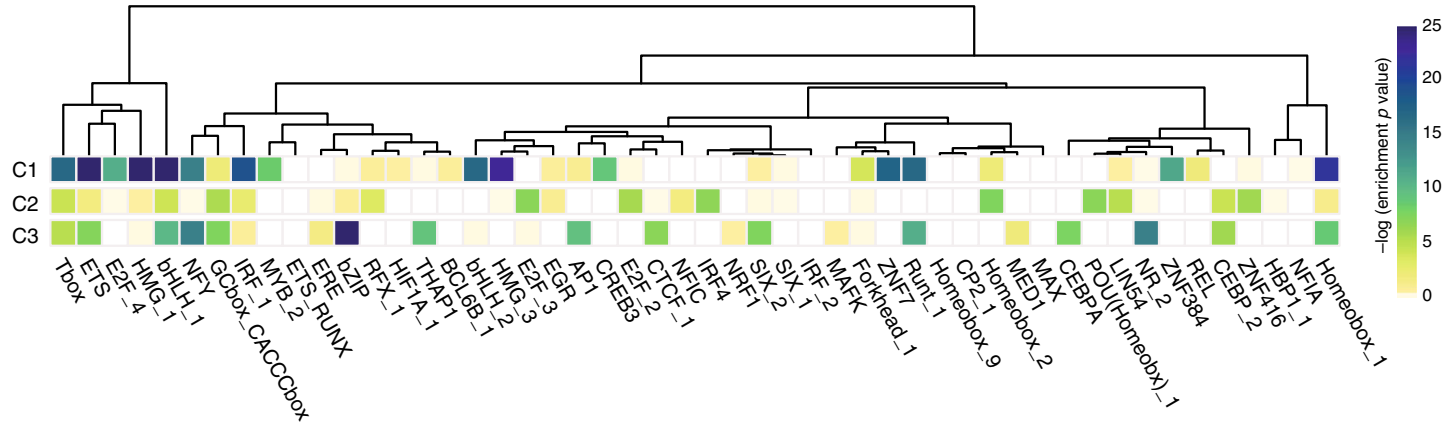

e Expression of TFs whose motifs are enriched in cluster $\mathrm{C} 1$

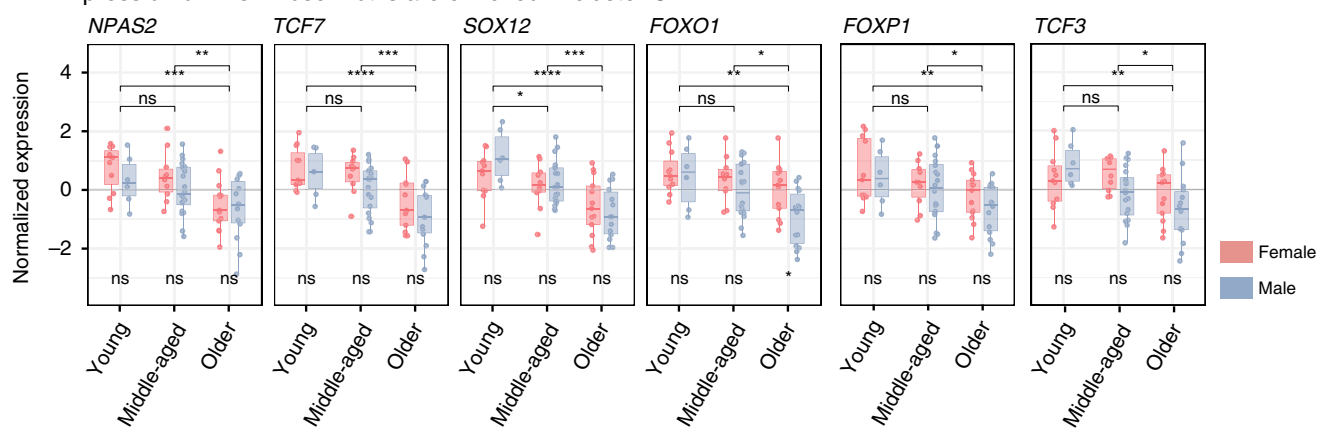

f Expression of TFs whose motifs are enriched in cluster $\mathrm{C}_{3}$

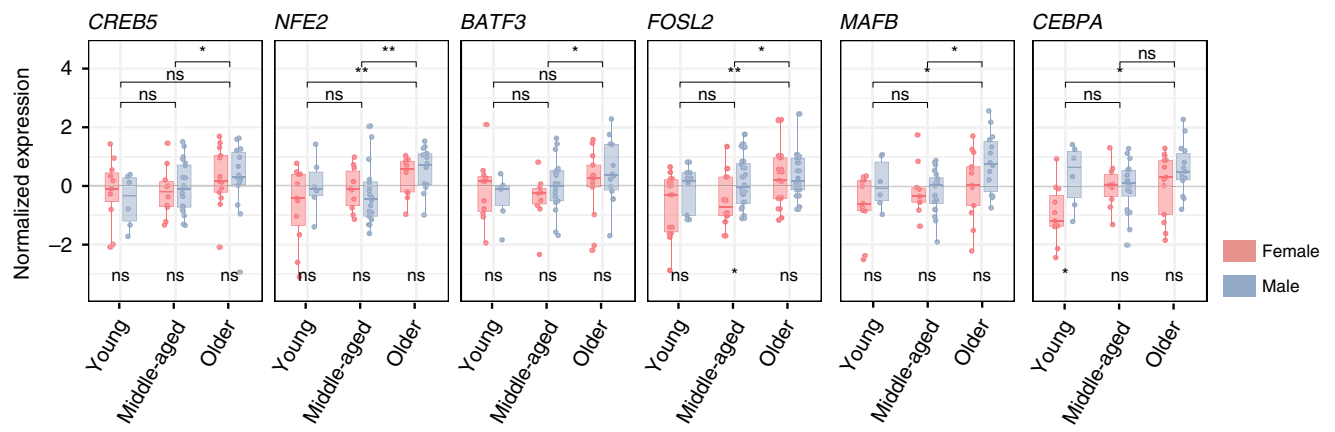

men, also leading to a significant difference (Wilcoxon $p=$ 0.0065) between older men and women (Fig. 6e).

These data suggest that older men show a bias towards innate immunity whereas older women towards adaptive immunity. This partially stems from the elevated activation of monocyte related genes/loci with age in men (Fig. 2c, Supplementary Fig. 4c). Fifteen times more monocyte-specific loci were activated in men with age (960 vs. 64 peaks). (Supplementary Fig. 2f). However, we did not detect significant sex differences in $\mathrm{CD} 14^{+}$ and $\mathrm{CD}_{16}{ }^{+}$monocyte cell numbers (counts and frequencies) in 
Fig. 5 Sex-specific patterns in temporal peaks/genes. a Heatmap of ATAC-seq peaks (fitted values from ARIMA models) with a chronological trend in both women and men $(n=3197)$, as a function of age in years. Values represent $z$-score normalized accessibility values relative to the row (i.e., peak) mean. K-means clustering was used to group these peaks into three clusters (C1-C3) using concatenated data from men and women. Color bar on the top represents discrete age groupings as defined in this study (young, middle-aged, older). Rows are annotated according to their position relative to the nearest TSS: proximal if $<1 \mathrm{kbp}$ distance, distal otherwise. b, c Annotations of shared temporal peaks using chromHMM states (b) and gene sets from DICE database $^{10}$ (c). Colors represent hypergeometric enrichment test $p$-values; light gray cells in plot indicate that there were insufficient genes in the gene sets to run an enrichment test. The enrichment pattern strongly associates cluster $\mathrm{C} 1$ to $\mathrm{T}$ cells, suggesting a delayed loss of accessibility in women relative to men; $\mathrm{C} 2$ to $\mathrm{CD} 19^{+}$cells, suggesting the presence of $\mathrm{CD} 19^{+}$specific loci with opposing temporal behavior in men and women; and C3 to monocytes and NK cells. d Transcription factor (TF) motif enrichment results for each temporal cluster (C1-C3), relative to the other two clusters. Motif enrichment analyses carried out on 1388 PWMs, grouped into families based on the sequence similarity, and most significant $p$-value for each motif family is represented here. Tests were done using HOMER ${ }^{54}$. e, f Expression levels of TFs associated to cluster 1 (C1) and cluster 3 (C3) grouped by age group and sex whose expression follows the same pattern as the peak temporal clusters where they are enriched. Cluster 2 (C2) is omitted since all TFs in this group show a significant increase with age in females or both sexes. Box plots represent median and IQR values, with whiskers extending to 1.5 times the IQR. Wilcoxon rank-sum test used to compare expression levels between sexes (significance value below boxes) and age groups (above boxes): ${ }^{\star} p<0.05,{ }^{\star \star} p<0.01$, ${ }^{\star \star \star} p<0.001$, ns: non-significant. Sample sizes for young individuals $n=11 \mathrm{~F}, 6 \mathrm{M}$, middle-aged $n=10 \mathrm{~F}, 20 \mathrm{M}$, older $n=13 \mathrm{~F}$, $14 \mathrm{M}$. Source data are provided as a Source Data file for $(\mathbf{a}, \mathbf{b}, \mathbf{d})$.

flow cytometry data from Milieu Intérieur Consortium ${ }^{12}(n=$ 892) (Supplementary Fig. 8d) or from our cohort (Fig. 1d). Even though the number of $\mathrm{CD} 16^{+}$monocytes increase with age in both sexes (Supplementary Fig. 8e), this increase was similar between sexes (Supplementary Fig. 8d, e). In contrast, the femalebias in adaptive immunity partially stemmed from male-specific genomic declines in B cell-associated loci (Figs. 2c, 4d) and B cell percentages within PBMCs (Fig. 1d). This trend was also observed in the Milieu Intérieur cohort for different B cell subsets (i.e., naive, transitional, founder) (Supplementary Fig. 8d, e). Interestingly, mass-cytometry data from a third $\operatorname{cohort}^{29}(n=$ 190 Caucasians) also revealed significant age-related declines in B cell percentages (Supplementary Fig. 8f). However, these declines were observed in both sexes. Further studies are needed to uncover whether B cell-related differences among cohorts can be due to clinical and/or environmental factors.

\section{Discussion}

Despite well-characterized sex differences in immune responses, disease susceptibility, and lifespan, it is unclear whether aging differentially affects peripheral blood cells of men and women. To fill this gap, we generated ATAC-seq, RNA-seq, and flow cytometry data in PBMCs from 172 age-matched healthy adults. Using novel systems immunology pipelines, we discovered a genomic signature of aging that is shared between sexes including (1) declines in T cell functions, (2) increases in cytotoxic (NK, memory $\mathrm{CD}^{+} \mathrm{T}$ ) and monocyte cell functions. This signature is in alignment with known age-related changes in the immune system including declining adaptive responses and increased systemic inflammation with age ${ }^{14,30}$. Despite these similarities, male PBMCs went through greater changes that are not attributable to clinical differences. Notably, 15 times more monocytespecific loci were activated in men compared to women. Previous studies in whole blood showed that DNA methylomes age faster in men compared to women ${ }^{31}$. Here, we uncovered an accelerated aging phenotype for men in ATAC-seq data and uncovered cell types, cellular functions, and molecules that differentially age between sexes. In addition, our data revealed for the first time that aging has opposing effects on the B cells of men and women, where B cell-specific loci/genes were modestly activated in women but significantly inactivated in men, which might contribute to sex-differences in auto-immunity and humoral responses.

We studied middle-aged individuals to capture chronological changes and the timing of changes, which is essential in distinguishing age-related changes from those resulting from maturation $^{32}$. Chronological patterns in ATAC-seq and RNA-seq data were correlated and included increasing accessibility/expression for cytotoxic cells and monocytes, and decreasing accessibility/ expression for $\mathrm{T}$ cells in both sexes. Temporal patterns from men and women further highlighted the differential aging of the B cellrelated loci. Breakpoint analyses uncovered that although agingrelated epigenomic changes accumulate gradually throughout adult life, there are two periods in the human lifespan during which the immune system undergoes abrupt changes. The first breakpoint was similar between sexes, however the second breakpoint was detected 5-6 years earlier in men, which is comparable to the life-span differences: 76.9 for men and 81.6 for women in $\mathrm{USA}^{33}$. In both sexes, the second breakpoint was associated with accelerated epigenomic changes and occurred 12-15 years before the end of the average lifespan. The differences in the timing of age-related changes can be helpful in clinical decisions regarding when to start interventions/therapies.

PBMCs of men and women significantly differed after the age of 65 , contrary to our expectations due to declining sex hormones. Annotation of sex-biased loci revealed that older women have higher genomic activity for adaptive cells and older men have higher activity for monocytes and inflammation. Some of these differences, but not all, are attributable to changes in cell compositions, e.g., the decline of B cell frequencies in older men (Fig. 1d), which is confirmed in a second cohort ${ }^{12}$ (Supplementary Fig. 8d) and has been reported in a Japanese cohort ${ }^{34}$. This concordance across cohorts suggests that sex-specific aging signatures described here might be conserved across ethnicities/ populations, which requires further investigation. Age-related increases were associated with inflammatory pathways/genes more significantly in men, suggesting an accelerated inflammaging signature for men. Data from an independent cohort ${ }^{11}$ supported this, where older men had higher levels of proinflammatory cytokines (IL6, IL18) in their serum compared to older women (Fig. 6e). Interestingly, we did not detect significant differences between older men and older women in $\mathrm{CD}_{1}{ }^{+}$and $\mathrm{CD} 6^{+}$monocyte cell numbers/frequencies neither in this cohort nor in a separate bigger cohort ${ }^{12}$ (Supplementary Fig. 8e). Therefore, age-related activation and sex-differences in monocytes potentially stems from cell-intrinsic changes. Future studies are needed to explore reasons behind sex differences in inflammaging, including the role of sex hormones and differential activation of DAMPs/PAMPs or transposable elements ${ }^{35}$.

Age-related changes in DNA methylation levels are predictive of ages across tissues and conditions, most notably the Horvarth's clock including $353 \mathrm{CpG}$ sites $^{36}$. A total of 187 (53\%) of these CpG sites overlapped PBMC ATAC-seq peaks $(86,145)$. The missing overlap likely stems from differences in technologies: CpG arrays vs. sequencing-based ATAC-seq assay 

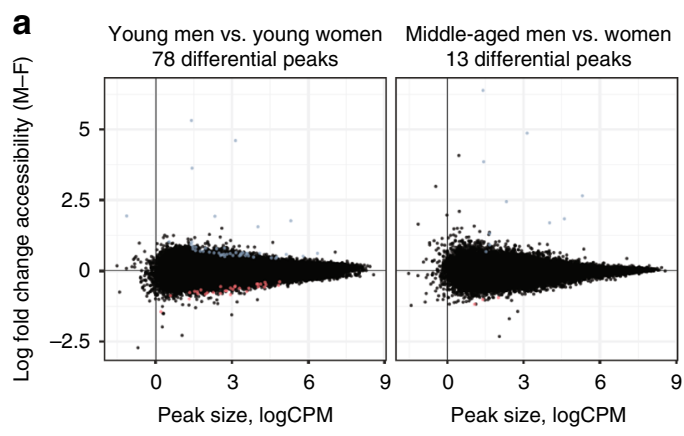

Older men vs. older women 1011 differential peaks
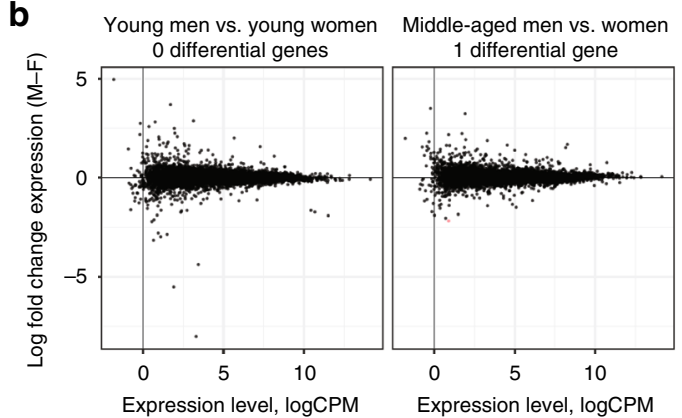

Expression level, logCPM

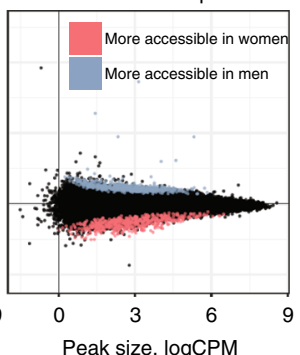

Older men vs. older women 548 differential genes

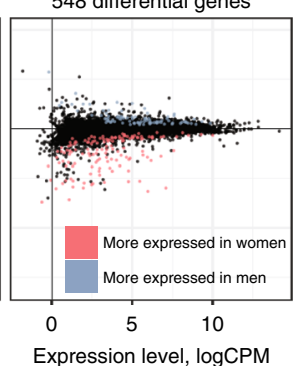

C Annotation of sex-biased loci

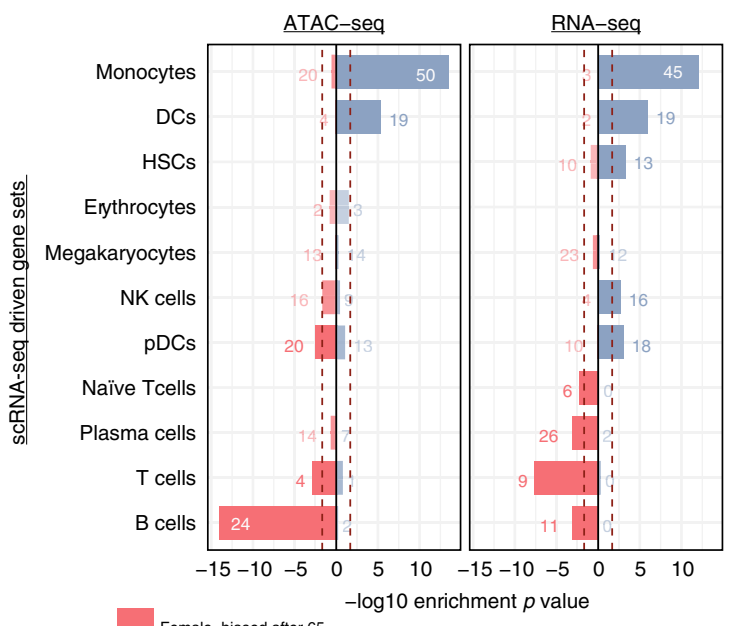

Female-biased after 65

Male-biased after 65

d

\begin{tabular}{|c|c|c|}
\hline Pathway/module name & FDR & No of hits/gene names \\
\hline IL-7 Signaling pathway (WP205) & 0.022 & 3/ JAK3, MYC, STAT5B \\
\hline PRC2 methylates, histones (WP3312) & $6.67 \mathrm{E}-49$ & 41/ HIST1H2AE, HIST1H2BD, HIST1H4I, HIST1H2AJ,... \\
\hline Interferon-inducible module* & 0.00068 & 7/ IFIT3, IFI44, PARP14, EIF2AK2, TNFSF10, C1GALT1, .. \\
\hline B cells module* & $1.61 \mathrm{E}-07$ & 9/ PLEKHG1, TCF4, GGA2, SNX22, PAX5, CD22, BLNK, ... \\
\hline NOD pathway (WP1433) & 0.027 & 3/ IL18, NAIP, RAC1 \\
\hline Inflammation II module & 2.93E-06 & 14/ H2AFY, CD302, MGST1, IL18, CPM, IRAK3, CD36, ... \\
\hline Inflammation I* & 0.0048 & 10/ TREM1, FAM49A, SMURF1, IFRD1, THBS1, SLA, MAFB,... \\
\hline Inflammation II module* & 0.00023 & 12/ CSF3R, CTSB, ASGR2, TFEB, RXRA, AOAH,LYN, CPM,... \\
\hline
\end{tabular}

\begin{tabular}{cc}
\multicolumn{2}{c}{ Changes with age } \\
\hline ATAC-seq & RNA-seq \\
\hline & NS,NS \\
& NS, NS \\
\hline$N S, N S$ & NS,NS \\
\hline
\end{tabular}

e

Pro-inflammatory cytokines and their receptor antagonists in serum ( $n=267,500 \mathrm{FG}$ project)
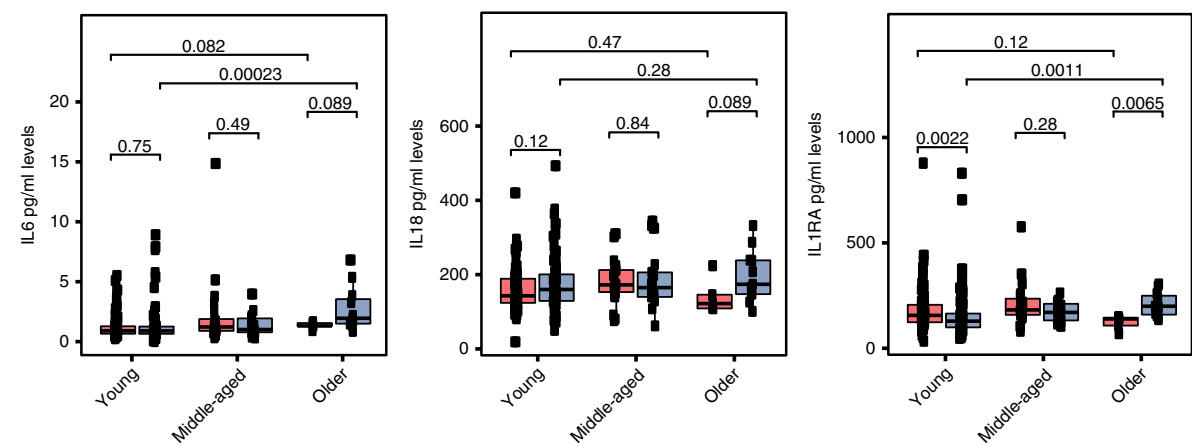

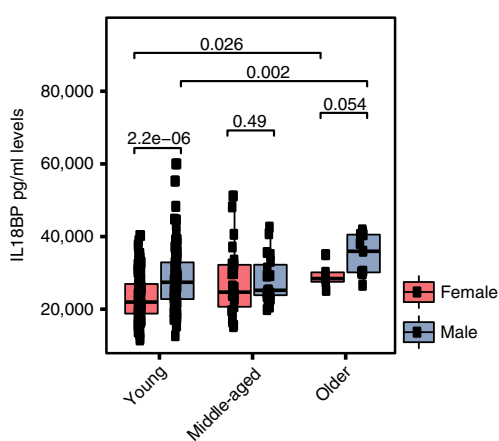

that genome-wide capture active regulatory elements ${ }^{37}$. Only a few CpGs in the clock overlapped opening/closing peaks (Supplementary Table 16), suggesting that ATAC-seq and DNA methylation arrays capture distinct epigenomic information and aging signatures. ATAC-seq captures age-related changes in regulatory element activity in a cell-specific manner. Although the mechanism underlying the $\mathrm{CpG}$ clocks are not established with certainty, they are proposed to represent widespread entropic decay of the DNA methylation landscape ${ }^{38}$.

Using a systems immunology approach in human PBMCs, this study uncovered cell types and immune functions that are differentially affected by aging between men and women. Changes in bulk PBMCs were annotated using cell-specific regulatory element loci inferred from reference epigenomic datasets ${ }^{10,20}$. Although this approach was effective in annotating the aging signatures, it is prone to biases in references, e.g., differences in data quality and limitation to use cell types available in references. Future studies are needed to describe these sex differences at single-cell resolution and in sorted cells and to establish their functional implications. Moreover, future studies are needed to study important molecules identified here (IL7R, LEF1, TCF7, IL8, IL18) as sexspecific biomarkers of immune system aging. Taken together, these findings indicate that sex plays a critical role in human immune system aging and should be taken into consideration while searching for molecular targets and time frames for interventions/therapies to target aging and age-related diseases. 
Fig. 6 Genomic differences between sexes at different age groups. MA plots representing mean log2 fold change (male-female) vs. average log2 read counts per million reads (logCPM) for ATAC-seq (a) and RNA-seq (b) data at three age groups using 78,167 peaks (12,199 genes) at autosomal chromosomes. Both epigenomic and transcriptomic differences between sexes increase with age. Peaks and genes significantly upregulated in women (men) relative to men (women) are represented in red (blue). Fold changes obtained via GLM modeling of the data sets; statistical significance assessed at a 5\% FDR threshold based on Benjamini-Hochberg $p$-value adjustment. Tests based on $n=100$ (ATAC-seq) and $n=74$ (RNA-seq) independent samples. (c) Enrichment of significantly sex-biased peaks/genes in older individuals using cell-specific gene sets obtained from single-cell RNA-seq data. Note the male bias toward increased accessibility/expression for monocytes and DCs and the female bias toward increased accessibility/expression for T and B cells. P-values are based on hypergeometric enrichment tests. Numbers on bars represent the number of differential genes overlapping each gene set. d Left: Selected pathways/module enrichments for male-biased and female-biased genes/loci. The complete list of enrichments is presented in Tables S12, S13. * represents annotations obtained from ATAC-seq data analyses. Right: Arrows indicating whether the same pathway/module has been significantly associated with age-related changes. Significant enrichments (FDR 5\%) and their directions are represented with arrows. Red arrows for women, blue arrows for men. NS = not significant. The complete list of enrichments is presented in Tables S6, S8. e ELISA data from 500 Functional Genomics (500FG) project to measure serum levels of pro-inflammatory cytokines $(n=267)$. Protein expression levels are compared between sexes and between young and older individuals. P-values were calculated using Wilcoxon rank sum (two sided). Box plots represent median and IQR values, with whiskers extending to 1.5 times the IQR. Source data are provided as a Source Data file for $(\mathbf{a}-\mathbf{c})$.

\section{Methods}

Human subjects. All studies were conducted following approval by the Institutional Review Board of UConn Health Center (IRB Number: 14-194J-3). Following informed consent, blood samples were obtained from 172 healthy volunteers residing in the Greater Hartford, CT, USA region recruited by the UConn Center on Aging Recruitment and Community Outreach Research Core (http://health. uconn.edu/aging/research/research-cores/). For older adults 65 years and older, recruitment criteria were selected to identify individuals who are experiencing "usual healthy" aging and are thus representative of the average or typical normal health status of the local population within the corresponding age groups ${ }^{5}$ Selecting this type of cohort is in keeping with the 2019 NIH Policy on Inclusion Across the Lifespan (NOT-98-024) ${ }^{39}$, increasing the generalizability of our studies and the likelihood that these findings can be translated to the general population ${ }^{40}$. Subjects were carefully screened in order to exclude potentially confounding diseases and medications, as well as frailty. Individuals who reported chronic or recent (i.e., within two weeks) infections were also excluded. Subjects were deemed ineligible if they reported a history of diseases such as congestive heart failure, ischemic heart disease, myocarditis, congenital abnormalities, Paget's disease, kidney disease, diabetes requiring insulin, chronic obstructive lung disease, emphysema, and asthma. Subjects were also excluded if undergoing active cancer treatment, prednisone above $10 \mathrm{mg}$ day, other immunosuppressive drugs, any medications for rheumatoid arthritis other than NSAIDs or if they had received antibiotics in the previous 6 months. Beyond these steps to exclude specific chronic conditions we also undertook further additional efforts to exclude older adults with any significant frailty. Since declines in self-reported physical performance are highly predictive of frailty, subsequent disability and mortality ${ }^{41}$, all subjects were also questioned as to their ability to walk $1 / 4$ mile (or $2-3$ city blocks). For those who self-reported an inability to walk $1 / 4$ mile $^{41}$, the "Timed Up and Go" (TUG) test was performed and measured as the time taken to stand up from the sitting position, walk 10 feet and return to sitting in the chair ${ }^{42}$. Scoring TUG $>10 \mathrm{~s}$ was considered an indication of increased frailty and resulted in exclusion from the study ${ }^{43}$. Information on medications in Table S1 illustrates that as expected medication usage did increase with age. Nevertheless, these medications all reflected their use for common and controlled chronic conditions unlikely to influence our findings such as hypertension, hyperlipidemia, hypothyroidism, degenerative joint disease, seasonal allergies, headaches, atrial fibrillation, depression, anxiety, or ADHD (attention deficit hyperactivity disorder). Finally, smoking history data are not typically collected in these studies-including ours-since smoking is a rare habit among older adults.

Ethics. The study was conducted following approval by the Institutional Review Board of UConn Health Center (IRB Number: 14-194J-3). All study participants provided written informed consent at baseline using institutional review boardapproved forms. Individual-level human genomic data (ATAC-seq and RNA-seq) are shared in a federal controlled-access database through dbGaP. Our study complies with Tier 1 characteristics for "Biospecimen reporting for improved study quality" (BRISQ) guidelines.

Flow cytometry data generation and analyses. PBMCs were isolated from fresh whole blood using Ficoll-Paque Plus (GE) density gradient centrifugation. For the analysis of the frequencies of Naive $\mathrm{T}$ cells $\left(\mathrm{CD} 45 \mathrm{RA}^{+} \mathrm{CCR} 7^{+}\right)$, Central Memory $\mathrm{T}$ cells $\left(\mathrm{CM} ; \mathrm{CD}^{2} \mathrm{RA}^{-} \mathrm{CCR} 7^{+}\right)$, Effector Memory T cells (EM; CD45RA $\left.{ }^{-} \mathrm{CCR} 7^{-}\right)$, and Effector Memory RA (EMRA; CD45RA ${ }^{+} \mathrm{CCR}^{-}$), B cells and Monocytes, PBMCs were stained with fluorochrome-labeled antibodies specific for $\mathrm{CD} 3$ (UCHT1) Biolegend-1:100 (cat\# 300436), CD4 (RPA-T4) Biolegend 1:80 (cat\# 558116), CD8 (SCFI21Thy2D3) Beckman Coulter 1:80 (cat\# 6604728), CD45RA (HI100) BD biosciences 1:80 (cat\# 560674), CD19 (HIB19) BD biosciences 1:100 (cat\#-555415), CD14 (MSE2) BD biosciences 1:80 (cat\# 557923), CCR7 (150503)
BD biosciences 1:20 (cat\# 561271). The stained cells were acquired with BD Fortessa and analyzed with FlowJo software (TreeStar). Flow data is analyzed using GLM to quantify the association between cell proportions and age, sex, and their interaction (age and sex). For the analyses of major cell populations, we used age (continuous variable) as a covariate, whereas for $\mathrm{T}$ cell subsets we used age group (old vs. young) as a variable. We excluded one outlier individual (subject 104) from downstream analyses.

ATAC-seq library generation and processing. ATAC-seq ${ }^{44}$ data was generated from fifty thousand unfixed nuclei using Tn5 transposase (Illumina, Nextera DNA sample prep kit) for $30 \mathrm{~min}$ at $37^{\circ} \mathrm{C}$. The resulting library fragments were purified using Qiagen MinElute kit (Qiagen). Libraries were amplified by 10-12 PCR cycles, purified using a Qiagen PCR cleanup kit (Qiagen), and finally sequenced on an Illumina HiSeq 2500 with a minimum read length of $75 \mathrm{bp}$ to a minimum depth of 30 million reads per sample. At least two technical replicates (average $=2.4$ replicates) were processed per biological sample. Table S2 summarizes the depth, peak number, and fragments in reads (FrIP) scores for ATAC-seq samples. ATACseq sequences were quality-filtered using trimmomatic ${ }^{45}$, and trimmed reads were mapped to the GRCh37 (hg19) human reference sequence using bwa-mem ${ }^{46}$. After alignment, technical replicates were merged and all further analyses were carried out on these merged data. For peak calling, MACS2 ${ }^{47}$ was used with no-model, $100 \mathrm{bp}$ shift, $200 \mathrm{bp}$ extension, and bampe option. Only peaks called with a peak score (q-value) of $1 \%$ or better were kept from each sample, and the selected peaks were merged into a consensus peak set using Bedtools multiinter tool ${ }^{48}$. Only peaks called on autosomal chromosomes were used in this study. We further filtered consensus peaks to avoid likely false positives by only including those peaks overlapping more than 20 short reads in at least one sample, and peaks for which the maximum read count did not exceed 500 counts per million (cpm) to account for regions that are potential artifacts. Finally, we excluded peaks overlapping ENCODE blacklisted regions downloaded from http://hgdownload.cse.ucsc.edu/ goldenpath/hg19/encodeDCC/wgEncodeMapability/. An additional quality-control step was developed to filter out samples with a consistently poor signal, consisting of an algorithm to discover and characterize a series of relatively invariant benchmark peaks, defined as a set of peaks expected to be called in all samples. Samples that consistently miss calls for a significant portion of these benchmark peaks are flagged as having poor quality. A benchmark peak is defined based on three criteria, namely (1) that it remains approximately invariant between the two groups of interest (i.e., young and old samples), (2) that it captures a substantial number of reads, and (3) that it is called in most samples. For each peak, the absolute value of the log of the ratio of healthy old to healthy young mean normalized read counts (log fold change, $\log \mathrm{FC}$ ) was used to assess the first criteria, whereas the maximum read count over all samples $(\operatorname{maxCt})$ is used to assess the second one. In this study, a peak was considered apt for benchmarking when (1) its absolute $\log \mathrm{FC}$ was in the bottom quartile of the distribution over all peaks, (2) its maxCt was in the top decile of the distribution over all peaks, and (3) the peak was called in at least $90 \%$ of the samples. Using these parameters, 640 (out of 86,300) peaks were selected as benchmark; only samples for which at least $92.5 \%$ of these peaks were called were selected for analyses, which excluded 8 samples from further analyses. We examined the effects of each of these parameter choices and found that the same samples were consistently chosen as poor quality for a range of values chosen to assess the benchmark criteria. After re-applying the peak selection criteria to the remaining 100 samples, we arrived at a peak count of 86,145 peaks. Among the samples that passed the QC step, we studied the distribution of FRIP scores, depth of sequencing, and peak numbers (that are correlated measures), which revealed a wide range of values. Neither of these measures were correlated significantly with age in linear regression models (FRIP: Pearson $r=0.11, p=0.29$; Depth: Pearson $r=0.081, p=0.42$; Peak number: Pearson $r=0.1, p=0.32$ ). However, on the average samples from men had higher values compared to samples from women (FRIP: 0.218 vs. 0.178; Depth: $103 \mathrm{M}$ vs. $72 \mathrm{M}$; Peak number 
37,046 vs. 29,170). To account for these differences, prior to statistical analyses, ATAC-seq read counts were normalized to each sample's effective library size (i.e., the sum of reads overlapping peaks) using the trimmed mean of M-values normalization method (TMM) ${ }^{49}$. In addition, we used the effective library size and significant surrogate variables as co-variates in differential analyses (see Differential analyses for details). Accordingly, we noted that SV1 in these analyses correlate significantly with library depth (Pearson $r=-0.68 p=6.8 \mathrm{e}-15$ ) and number of peaks (Pearson correlation $r=-0.31, p=0.0016$ ) in old-young comparisons and with library depth (Pearson $r=-0.56, p=1.7 \mathrm{e}-09$ ) and FRIP score (Pearson $r=$ $0.2, p=0.044)$ in men-women comparisons.

\section{RNA-seq library generation and processing. Total RNA was isolated from} PBMCs using the Qiagen RNeasy (Qiagen) or Arcturus PicoPure (Life Technologies) kits following manufacturer's protocols. During RNA isolation, DNase I treatment was additionally performed using the RNase-free DNase set (Qiagen). RNA quality was checked using an Agilent 2100 Bioanalyzer instrument, together with the 2100 Expert software and Bioanalyzer RNA 6000 pico assay (Agilent Technologies). RNA quality was reported as a score from 1 to 10 , samples falling below threshold of 8.0 being omitted from the study. cDNA libraries were prepared using either the TruSeq Stranded Total RNA LT Sample Prep Kit with Ribo-Zero Gold (Illumina) or KAPA Stranded mRNA-Seq Library Prep kit (KAPA Biosytems) according to the manufacturer's instructions using $100 \mathrm{ng}$ or $500 \mathrm{ng}$ of total RNA. Final libraries were analyzed on a Bioanalyzer DNA 1000 chip (Agilent Technologies). Paired-end sequencing $(2 \times 100 \mathrm{bp})$ of stranded total RNA libraries was carried out in either Illumina NextSeq500 using v2 sequencing reagents or the HiSeq2500 using SBS v3 sequencing reagents. Quality control (QC) of the raw sequencing data was performed using the FASTQC tool, which computes read quality using summary of per-base quality defined using the probability of an incorrect base call ${ }^{50}$. According to our quality criteria, reads with more than $30 \%$ of their nucleotides with a Phred score under 30 are removed, whereas samples with more than $20 \%$ of such low-quality reads are dropped from analyses. Benchmarking is also applied on RNA-seq data using the same benchmark parameters as ATAC-seq, which resulted in 304 benchmark genes, none of the RNA-seq samples were dropped due to poor quality. Reads from samples that pass the quality criteria were quality-trimmed and filtered using trimmomatic ${ }^{45}$. High-quality reads were then used to estimate transcript abundance using RSEM ${ }^{51}$. Finally, to minimize the interference of non-messenger RNA in our data, estimate read counts were renormalized to include only protein-coding genes. Table S2 summarizes the quality control measures for our PBMC RNA-seq samples.

Differential analysis. To identify differentially open chromatin regions from ATAC-seq and differentially expressed genes from RNA-seq data, the R package edgeR was used to fit a GLM to test for the effect of aging between healthy young and healthy old samples by sex, as well as the effect of sex by age group. In addition to sex and age group (old vs. young), our models included the base-2 $\log$ of effective library size to ensure peakwise normalization. We isolated a batch effect correlated to time period whereby samples were collected and libraries were prepared, and used ComBat to adjust the data for this effect. Finally, we used surrogate variable analysis $\left(\mathrm{SVA}^{52}\right)$ to capture unknown sources of variation (e.g., localized batch effects, subject-level heterogeneity, variation in library preparation techniques) statistically independent from age group assignments. SVA decomposes the variation that is not accounted for by known factors like age group or sex, into orthogonal vectors that can then be used as additional covariates when fitting a model to test for differential accessibility or expression. Using the built-in permutation-based procedure in the R package sva, we choose to retain one SV to include as covariate in the GLM model for PBMC ATAC-seq and none for RNAseq data analyses ${ }^{53}$. GLM models where implemented using a negative binomial link function, including both genome-wide and peak-specific dispersion parameters, estimated using edgeR's "common," "trended," and "tagwise" dispersion components, calculated using a robust estimation option. Benjamini-Hochberg $p$-value correction was used to select differentially open peaks at a false discovery rate (FDR) of $5 \%$. To generate a set of model-adjusted peak estimates of chromatin accessibility (i.e., batch-, and SV-adjusted) for downstream analyses and visualization, we used edgeR to fit a "null" model excluding the sex and age group factor, and then subtracted the resulting fitted values from this model from the original TMM-normalized reads.

Peak annotation and downstream analyses. Multiple data sources were used to annotate ATAC-seq peaks with regard to functional and positional information. HOMER $^{54}$ was used to annotate peaks as "promoter" (i.e., within $2 \mathrm{~kb}$ of known TSS), "intergenic", "intronic", and other positional categories. For functional annotation of peaks, we used a simplified scheme integrating public chromatin states calculated for major PBMC subpopulations with ChromHMM from Roadmap Epigenomics ${ }^{20}$, Blueprint Epigenome, and a third reference data ${ }^{55}$ : First, we intersected the chromHMM-generated states with our set of consensus peaks, and solved conflicting cases where multiple chromatin states overlap the same ATACseq peak so that each peak was assigned a single annotation, according to the following priority rules: if a peak overlaps both an active TSS and enhancer region, which state takes priority depends on whether the peak is proximal (i.e., within
$1000 \mathrm{bp}$ of the nearest TSS), in which case it is annotated as a promoter, or distal (distance to nearest TSS greater than $1000 \mathrm{bp}$ ), when it is annotated as an enhancer instead. For all other states, rules apply as follows: Active Enhancer $>$ Genic Enhancer $>$ Bivalent TSS $>$ Weak Enhancer $>$ Bivalent Enhancer $>$ PolyComb repressed $>$ TSS Flanking $>$ Transcription $>$ ZNF Genes and repeats $>$ Heterochromatin $>$ Quiescent/Low signal. Then, to facilitate interpretation and visualization, we simplified the original sets of chromatin states to a scheme with 6 pooled meta-states, namely (1) TSS, collecting active, flanking, and bivalent TSS states; (2) Enhancer, pooling active, weak and bivalent enhancer states; (3) Repressed PolyComb, combining both weak and strong PolyComb states; (4) Transcription, including both weak and strong transcription states, (5) the quiescent chromHMM state; and (6) other states (ZNF, heterochromatin) combined together. To annotate peaks as cell-specific for a given subset obtained from one of the three datasets listed above, we determined for each peak whether it was annotated as an active promoter or an active enhancer in a single-cell population or lineage, and in such cases labeled the peak accordingly as cell- or lineage specific. For example, if a peak is annotated as an active enhancer in both naive and memory $\mathrm{CD}^{+} \mathrm{T}$ cells but as another state (e.g., repressed) in every other subset, then the peak would be considered $\mathrm{CD}_{4}^{+} \mathrm{T}$-specific. For gene-based analyses, HOMER was used to assign each ATAC-seq peak to the nearest TSS, as measured from the peak center. To improve confidence on these annotations, gene-based analyses were further restricted to include only peaks located within $100 \mathrm{~kb}$ of their corresponding TSS. ATAC-seq peaks were also annotated using gene sets provided by curated immune function-related co-expression modules ${ }^{13}$. These gene sets comprise 28 modules defined from multiple compiled transcriptomic data sets, which were originally annotated based on expert knowledge of representative functions of the gene ensemble in each module. In this study, we have preserved and used these annotations to test for enrichment of these modules in gene sets of interest, such as the set of genes associated to chromatin peaks gaining or losing accessibility with aging We assessed enrichment using the hypergeometric test followed by BenjaminiHochberg FDR adjustment for P-values. Further functional enrichment analyses were carried out using Wikipathways pathways ${ }^{56}$, immune modules ${ }^{22}$, gene sets from DICE database ${ }^{10}$ and single-cell RNA-seq data in PBMCs. Gene sets from PBMC scRNA-seq data is driven from one-vs-all cell cluster comparisons. First, we identified 17 clusters from PBMC scRNA-seq data ( $n=26$ samples) using the Louvain clustering in the ScanPy toolkit ${ }^{57}$. These clusters were manually inspected and assigned to different cell types by studying the expression of known marker genes. For each cluster, differentially expressed genes were identified using one-vsall approach based on T-test followed by FDR. Marker genes for a cluster (or cell population) were found by using FDR $\leq 5 \%$ and $\log \mathrm{FC}>\log 2(1.25)$ using differential analyses results. Similarly, marker genes from the DICE database are obtained using their differential analyses results and the same cutoffs for cellspecific genes (FDR $\leq 5 \%$ and $\log F C>\log 2(1.25)$ ). Gene sets used in our differential analyses are provided as a supplementary table (Table S14). Similarly, peak sets from ChromHMM states used in the enrichment analyses are provided as a supplementary table (Table S15). For each gene set, we tested for enrichment using the hypergeometric test, against a background defined by the set of genes that are expressed, as determined by RNA-seq data, or potentially expressed, as given by promoter accessibility, in PBMCs. We used the Benjamini-Hochberg FDR multiple test correction to assess significance of hypergeometric $p$-values.

ATAC-seq and RNA-seq comparisons. Gene expression (RNA-seq, see above) data were generated for a subset of subjects with ATAC-seq profiles (summarized in Table S1). These data were normalized to protein-coding transcripts, and annotated to ENSEMBL GRCh37 gene symbols. Genes for which at least three normalized reads per million were obtained in at least two samples were considered as expressed, all others removed prior to analysis. This resulted in a total estimate of 14,157 expressed genes in PBMCs. We built a data set comprising paired ATACseq and RNA-seq samples by matching promoter peaks to nearest gene (TSS) annotations, defining promoters as the regions within $1000 \mathrm{bp}$ flanks of each TSS. Whenever multiple expressed genes were matched to the same promoter peak, the peak with the maximum fold change was chosen for visualization.

Inferring chronological aging trends. We used ARIMA models as implemented in $\mathrm{R}$ on age-ordered normalized chromatin accessibility and expression data separately for each sex, to select genomic features (ATAC-seq peaks or mRNA-seq transcripts) to uncover significant chronological trends, as opposed to unstructured "noise" fluctuations undistinguishable from stationary data. Input data from all subjects in the study (see Table S1) were normalized as described above in "Differential Analyses", i.e., corrected for known batch effects using ComBat, and model-adjusted to correct for library size and unknown batch effects (i.e., surrogate variables) using EdgeR. Thus, ARIMA-modeled data contains variation due to age, sex, and error variance unaccounted for by batch and library size effects. For model fitting, genomic features were converted into z-ordered objects (using R package zoo) after averaging values obtained for the same sex and chronological age (in years), and into time series objects. Formatted time series were then analyzed using auto.arima (from the forecast $\mathrm{R}$ package), using stepwise search and Akaike Information Criterion (AIC) to select the best model. This algorithm scans a wide range of possible ARIMA models and selects the one that satisfies the optimality criterion, i.e., the smallest AIC. Only non-seasonal models with a maximum 
difference $(D)$ of 2 were considered in this study, such that a non-stationary trend the data can be modeled after integrating a stationary series once or twice. Overall, best-fitting ARIMA models based on chromatin accessibility or gene expression data included models with 0-7 AR, 0-5 MA, and 0-7 AR + MA parameters for accessibility and 0-4 AR, 0-3 MA, and 0-4 AR + MA parameters for expression. From these models, we chose for inspection only the ones carrying a "significant" trend, defined as those where (1) the difference order from stationary was higher than zero, and (2) at least one AR or MA coefficient was included in the model (i.e., $D>0 \cap \mathrm{AR}+\mathrm{MA}>0$ ). These criteria allow sampling a wide variety of trend patterns if they were present in the data. For accessibility data, 14,594 (18.7\%) and $13,591(17.4 \%)$ peak models had $D=1$ (none had $D=2)$ in females and males respectively, out of which 13,297 (17\%) and 13,295 (17\%), respectively, also were estimated to have AR $+\mathrm{MA}>0$, and hence chosen for further analyses. Out of the chosen peaks, 12,941 and 11,566 had only MA (9082 in females, 8696 in males) or AR (2,562 in females, 2574 in males) coefficients, whereas only $4196(31.6 \%)$ and $4,181(31.4 \%)$ were estimated to have two or more non-zero (AR + MA) coefficients in females and males, respectively. A total of 3197 (4.1\%) peaks were chosen in both sexes, whereas the remaining peaks were treated as sex-specific. For expression data, $1367(11.1 \%)$ and 1665 (13.5\%) models had $D=1$ (none had $D=$ 2 ) in females and males respectively, out of which 1068 (8.6\%) and $1471(11.9 \%)$, respectively, also were estimated to have $\mathrm{AR}+\mathrm{MA}>0$, and hence used in further analyses. Out of these, 1359 and 832 had only MA (511 in females, 903 in males) or AR (480 in females, 372 in males) coefficients, whereas only $503(36.8 \%)$ and 554 (33.3\%) were estimated to have two or more non-zero (AR + MA) coefficients in females and males, respectively. A total of $180(1.5 \%)$ peaks were chosen in both sexes, whereas the remaining peaks were treated as sex-specific.

Temporal peak/gene analyses. After selecting genomic features with a significant trend, we used their estimated parameters to fit the original data to reduce noise and facilitate the discovery of common trend patterns across multiple features. Fitted values were computed at the same chronological ages observed in the data for each sex, converted to $z$-scores for comparability, and then submitted to $k$ means clustering for aggregation into $k$ sets of similar chronological aging patterns. Chosen features for each sex were clustered separately, and additionally features chosen as common to both sexes were concatenated as a single sequence of two age-ordered ages prior to normalization. This concatenation approach was designed to facilitate the detection of features with consistent chronological patterns across sexes. For clustering, we used the R-stats version of the $k$-means algorithm (kmeans), starting from nstart $=1000$ random sets of $k$ cluster centers and running each for max.iter $=100$ iterations. To choose $k$, we opted for a biologically informed criterion as opposed to a purely statistical one, after noting that criteria such as maximization of within- vs. between-cluster variance tended to select a large $(>20)$ number of slightly different clusters, likely emphasizing small differences in ARIMA model parameters with little biological significance. Instead, we computed optimal clusters for values of $k$ between 2 and 15 and calculated enrichment statistics for cell-specific features (i.e., cell-specific chromatin states for ATAC-seq, and cell-specific expressed genes for RNA-seq data) for each cluster relative to all trending features, and asked whether adding clusters to the $k=2$ cluster case resulted in a gain of information demonstrated by cluster enrichment patterns qualitatively different from the patterns observed in absence of the extra clusters. In some cases, finding a cluster configuration satisfying this condition of biological discrimination required applying hierarchical clustering to collapse distinct $k$-means clusters with the same biological signal. For chromatin accessibility, conservative application of these criteria led to the selection of $k=5$ and $k=$ 6 clusters for females and males, respectively, which were collapsed into three clusters in both cases (one closing, two opening with aging), and $k=3$ for peaks trending in both sexes (one closing and one opening in both sexes, plus one opening in females while closing in males). The same numbers of final clusters were defined based on gene expression.

TF motif enrichment analyses. To further explore functional associations of these clusters, we tested for enrichment of known and de novo TF motifs in each chromatin accessibility cluster for female, male, and sex-pooled peak data, relative to all trending clusters for the same set of peaks. We used the software HOMER ${ }^{54}$ to both detect de novo motifs and to test for enrichment of 1388 motifs obtained from JASPAR CORE 2018 databas $e^{58}$ and from Jolma et al. ${ }^{59}$ SELEX-derived position weight matrices (PWMs), corresponding to 680 distinct TFs. We used HOMER to assign a TF to each de novo motif found, based on sequence similarity to the 1388 known motifs. From the pooled set of known and de novo motifs tested for each cluster, we used Benjamini-Hochberg method on enrichment $p$-values to estimate FDR, and applied a 5\% FDR significance threshold to select for the final set of enriched motifs. Since some motifs have similar consensus sequences and PWMs, prior to visualization of these results we grouped motifs into "families" defined as sets of motifs with nearly identical sequences, as determined by pairwise comparison of all 1388 motif using MEME/TOMTOM ${ }^{60}$, which tests whether two motif sequences are more different than expected by chance. Using stringent $0.001 \% q$-value and $5 \mathrm{E}$-value cutoffs, we identified 429 motif families with extremely similar consensus sequences, 319 of which were singletons (maximum family size $=226$ motifs).
Breakpoint analyses. We investigated systemic chronological signatures in temporal peaks by testing, in each cluster, for the existence of "breakpoints," i.e., short age intervals characterized by significant differences in accessibility in the intervals preceding and following the age interval. For each age $t$ in the sampled age interval from $t_{\min }$ to $t_{\max }$, we tested for mean difference in accessibility between subjects with ages in the intervals $t_{\min }-w$ vs. $t_{\min }+w$, where $w$ represents a variable window span parameter, and plotted the observed $p$-values (i.e., $-\log 10 \mathrm{P}$, or $\operatorname{login} v p$ ) as a function of age $(t)$ to identify maxima that suggested the presence of discrete breakpoints. These tests were carried out on normalized and model-adjusted accessibility data corresponding to the ATAC-seq peaks associated to each sexspecific and common cluster identified as trending by ARIMA as described above. Since there were many more peaks than subjects for any given comparison, we used PCA to reduce the dimensionality of each cluster to $n=3$ PCs, and used MANOVA on these three dimensions to compute $p$-values at each tested value of $t$. For any given value of $w$, offset values for $t_{\min }$ and $t_{\max }$ were adjusted to match the age of available samples in the study. For example, a window span of 5 years required a $t_{\min }=27$ if the youngest available subject was 22 years old, and a $t_{\max }=83$ if the oldest available subject was 90 years old. For a given value of $w$, results from tests contrasting younger vs. older intervals would vary depending on sample size volume and imbalance, with statistical power increasing with the size of the window span. To take advantage of this effect, we deployed a multi-scale algorithm where we carried out tests using $w$ values ranging from 10 to 20 years in order to identify breakpoints that were maximally supported under multiple window spans. Due to sample sparsity and variation, however, tests carried out under varying values of $w$ may be unevenly affected by edge effects and influencing outlier points, which may result in strong significance of a comparison because of the presence of outliers and the partial overlap of a sampled interval with a breakpoint, limiting the ability of the method to precisely discover where such breakpoints may lie. To limit these effects and increase the robustness of the tests, we smoothed the loginvp distributions by fitting LOESS regressions to each comparison (i.e., each set of tests with the same $w$ value) under a range of smoothing bandwidth parameters (i.e., $\mathrm{bw}=0.25,0.30, \ldots, 0.70,0.75)$. We combined the resulting $11 p$-values at every sampled age using the Fisher's method, reapplied LOESS smoothing to the resulting distribution, and used numerical differentiation to determine whether each age was predicted to be minimum or a maximum. Finally, we marked every maximum as a significant breakpoint candidate if it satisfied both a parametric criterion, i.e. significance of the Fisher method-combined $p$-values ( $\chi^{2}$ test), and a heuristic criterion, namely whether the distance between this local maximum and the nearest minimum equaled or exceeded $25 \%$ of the value of the global maximum. The procedure described above results in a smoothed loginvp distribution for each $w$ value, each comprising a series of points including maxima and minima, such that slightly different maxima can be estimated for different $\mathrm{w}$ values. Finally, we used Gaussian mixture modeling on the distribution of these maxima, as implemented in R-Mclust package, to group loginvp maxima obtained from different window spans into cohesive breakpoint intervals, whose medians and ranges we report herein for each cluster. Since breakpoints are independently calculated for each cluster, observed overlaps are likely the result of aging-related events with a genome-wide impact.

Analyses of 500FG and MI data. We obtained publicly available ELISA data measuring serum protein levels by the 500 Human Functional Genomics (500FG) consortium ${ }^{11}$. We only retained individuals who are matching our cohort in terms of the age span, which resulted in data from 267 individuals. These individuals are grouped together using the age brackets defined in our study: Young 22-40 years old, middle-aged: 41-64 years old, older: 65 years old. We compared data from (1) men and women at all age brackets; (2) young men to old men; and (3) young women to old women using Wilcoxon Rank Sum non-parametric test (two sided) Note that flow cytometry data from this same cohort was not publicly available; hence cannot be included into the analyses. Similarly, publicly available flow cytometry data from Milieu Intérieur Consortium ${ }^{12}$ cohort was obtained. We used data that is already processed by this study and just used individuals whose ages are matching our cohort, which resulted in data from 892 individuals. We built linear models using $\mathrm{R}$ ( $\mathrm{lm}$ function) to quantify the association between each flow cytometry measurement to age group (young, middle-aged, older), sex (F, M) and their interaction (age*sex). Significant associations with age (at FDR 5\%) and with sex (at FDR 10\%) are plotted in Figs. S7D-E. No significant associations were detected with the interaction of sex and age at FDR $10 \%$.

R Shiny application. The R Shiny package ${ }^{61}$ was used to create a webpage for the interactive visualization of the data from this study. Plots were generated using ggplot $2^{62}$, using graph esthetics used throughout the manuscript figures. Statistics for box plots were calculated using the wilcox.test function in R and presented without correction for the multiple $(n=5)$ comparisons (two sided). Statistics for scatterplots were calculated by fitting a linear model with the $\mathrm{lm}$ function in $\mathrm{R}$ using the formula (measurement $\sim$ age:sex + sex). For ATAC-seq data, only the peak closest to the gene TSS was considered.

Reporting summary. Further information on research design is available in the Nature Research Reporting Summary linked to this article. 


\section{Data availability}

The data generated as part of this study is controlled access. A subset of the ATAC-seq and RNA-seq samples used in these analyses was made public through EGA (Id: EGAS00001002605). All PBMC ATAC-seq and RNA-seq samples used in this study can be found at dbGaP (Id: phs001934.v1.p1). The source data underlying Figs. 1c, 2a-c, e, $3 \mathrm{a}-\mathrm{d}, 4 \mathrm{a}-\mathrm{e}, 5 \mathrm{a}, \mathrm{b}, \mathrm{d}$, and $6 \mathrm{a}-\mathrm{c}$ and Supplementary Figs. 1c, g, 2b-e, 4a, c, d, g, 5a-c, 6a, b, $7 \mathrm{a}-\mathrm{c}, 8 \mathrm{a}-\mathrm{c}$ are provided as a Source Data file.

\section{Code availability}

The code is available at (https://github.com/UcarLab/SexDimorphismNatureCommunications). The R Shiny app is publicly shared at (https://immune-aging.jax.org/). Code for the R Shiny app is available at https://github.com/TheJacksonLaboratory/Ucar_Aging_Shiny.

Received: 13 August 2019; Accepted: 3 January 2020;

Published online: 06 February 2020

\section{References}

1. Castelo-Branco, C. \& Soveral, I. The immune system and aging: a review. Gynecol. Endocrinol. 30, 16-22 (2014).

2. Peters, M. J. et al. The transcriptional landscape of age in human peripheral blood. Nat. Commun. 6, 8570 (2015).

3. Jones, M. J., Goodman, S. J. \& Kobor, M. S. DNA methylation and healthy human aging. Aging Cell 14, 924-932 (2015).

4. Moskowitz, D. M. et al. Epigenomics of human CD8 T cell differentiation and aging. Sci. Immunol. 2, eaag0192 (2017).

5. Ucar, D. et al. The chromatin accessibility signature of human immune aging stems from CD8(+) T cells. J. Exp. Med. 214, 3123-3144 (2017)

6. Giefing-Kröll, C., Berger, P., Lepperdinger, G. \& Grubeck-Loebenstein, B. How sex and age affect immune responses, susceptibility to infections, and response to vaccination. Aging Cell 14, 309-321 (2015).

7. Klein, S. L. \& Flanagan, K. L. Sex differences in immune responses. Nat. Rev. Immunol. 16, 626 (2016).

8. Abdullah, M. et al. Gender effect on in vitro lymphocyte subset levels of healthy individuals. Cell. Immunol. 272, 214-219 (2012).

9. Fan, H. et al. Gender differences of B cell signature in healthy subjects underlie disparities in incidence and course of SLE related to estrogen. J. Immunol. Res. 2014, 814598 (2014)

10. Schmiedel, B. J. et al. Impact of genetic polymorphisms on human immune cell gene expression. Cell 175, 1701-1715.e1716 (2018).

11. Bakker, O. B. et al. Integration of multi-omics data and deep phenotyping enables prediction of cytokine responses. Nat. Immunol. 19, 776 (2018).

12. Piasecka, B. et al. Distinctive roles of age, sex, and genetics in shaping transcriptional variation of human immune responses to microbial challenges. Proc. Natl Acad. Sci. USA 115, E488-E497 (2018).

13. Chaussabel, D. et al. A modular analysis framework for blood genomics studies: application to systemic lupus erythematosus. Immunity 29, 150-164 (2008).

14. Goronzy, J. J. \& Weyand, C. M. Understanding immunosenescence to improve responses to vaccines. Nat. Immunol. 14, 428-436 (2013).

15. Kleiveland, C. R. In: (eds Verhoeckx, K., Cotter, P., López-Expósito, I., Kleiveland, C., Lea, T., Mackie, A., Requena, T., Swiatecka, D. \& Wichers, H.) The Impact of Food Bioactives on Health (eds). (Springer, Cham, 2015).

16. Patin, E. et al. Natural variation in the parameters of innate immune cells is preferentially driven by genetic factors. Nat. Immunol. 19, 302 (2018).

17. Olson, N. C. et al. Decreased naive and increased memory CD4 $(+)$ T cells are associated with subclinical atherosclerosis: the multi-ethnic study of atherosclerosis. PLoS ONE 8, e71498 (2013).

18. Clave, E. et al. Human thymopoiesis is influenced by a common genetic variant within the TCRA-TCRD locus. Sci. Transl. Med. 10, eaao2966 (2018).

19. Robinson, M. D., McCarthy, D. J. \& Smyth, G. K. edgeR: a Bioconductor package for differential expression analysis of digital gene expression data. Bioinformatics 26, 139-140 (2010).

20. Kundaje, A. et al. Integrative analysis of 111 reference human epigenomes. Nature 518, 317 (2015).

21. Feser, J. \& Tyler, J. Chromatin structure as a mediator of aging. FEBS Lett. 585, 2041-2048 (2011)

22. Chaussabel, D. \& Baldwin, N. Democratizing systems immunology with modular transcriptional repertoire analyses. Nat. Rev. Immunol. 14, 271 (2014).

23. Hidalgo, L., Einecke, G., Allanach, K. \& Halloran, P. The transcriptome of human cytotoxic $\mathrm{T}$ cells: similarities and disparities among allostimulated CD4+ CTL, CD8+ CTL and NK cells. Am. J. Transplant. 8, 627-636 (2008).

24. Wang, S. et al. S100A8/A9 in Inflammation. Front. Immunol. 9, 1298 (2018).
25. Brockwell, P. J., Davis, R. A. \& Calder, M. V. Introduction to Time Series and Forecasting. (Springer, 2002).

26. Wöhner, M. et al. Molecular functions of the transcription factors E2A and E2-2 in controlling germinal center B cell and plasma cell development. J. Exp. Med. 213, 1201-1221 (2016).

27. Kijima, M. et al. Dendritic cell-mediated NK cell activation is controlled by Jagged2-Notch interaction. Proc. Natl Acad. Sci. USA 105, 7010-7015 (2008)

28. Johnson, J. L. et al. Lineage-determining transcription factor TCF-1 initiates the epigenetic identity of T cells. Immunity 48, 243-257. e210 (2018).

29. Whiting, C. C. et al. Large-scale and comprehensive immune profiling and functional analysis of normal human aging. PLoS ONE 10, e0133627 (2015).

30. Fulop, T. et al. Immunosenescence and inflamm-aging as two sides of the same coin: friends or foes? Front. Immunol. 8, 1960 (2018).

31. Hannum, G. et al. Genome-wide methylation profiles reveal quantitative views of human aging rates. Mol. Cell 49, 359-367 (2013).

32. Coleman, P., Finch, C. \& Joseph, J. The need for multiple time points in aging studies. Neurobiol. Aging 11, 1-2 (1990).

33. World Health Organization. World Health Statistics 2016: Monitoring Health for the SDGs Sustainable Development Goals. (World Health Organization, 2016).

34. Hirokawa, K. et al. Slower immune system aging in women versus men in the Japanese population. Immun. Ageing 10, 19 (2013).

35. De Cecco, M. et al. L1 drives IFN in senescent cells and promotes ageassociated inflammation. Nature 566, 73 (2019).

36. Horvath, S. DNA methylation age of human tissues and cell types. Genome Biol. 14, 3156 (2013).

37. Buenrostro, J. D., Giresi, P. G., Zaba, L. C., Chang, H. Y. \& Greenleaf, W. J. Transposition of native chromatin for multimodal regulatory analysis and personal epigenomics. Nat. Methods 10, 1213 (2013).

38. Field, A. E. et al. DNA methylation clocks in aging: categories, causes, and consequences. Mol. cell 71, 882-895 (2018).

39. Kuchel, G. A. Inclusion of older adults in research: ensuring relevance, feasibility, and rigor. J. Am. Geriatrics Soc. 67, 203-204 (2019).

40. Robertson, D. \& Williams, G. H. Clinical and Translational Science: Principles of Human Research. (Academic Press, 2009)

41. Hardy, S. E., Kang, Y., Studenski, S. A. \& Degenholtz, H. B. Ability to walk 1/4 mile predicts subsequent disability, mortality, and health care costs. J. Gen. Intern. Med. 26, 130-135 (2011).

42. Podsiadlo, D. \& Richardson, S. The timed "Up \& Go": a test of basic functional mobility for frail elderly persons. J. Am. Geriatrics Soc. 39, 142-148 (1991)

43. Rockwood, K., Awalt, E., Carver, D. \& MacKnight, C. Feasibility and measurement properties of the functional reach and the timed up and go tests in the Canadian study of health and aging. J. Gerontol. Ser. A, Biol. Sci. Med. Sci. 55, M70-M73 (2000)

44. Buenrostro, J. D., Giresi, P. G., Zaba, L. C., Chang, H. Y. \& Greenleaf, W. J. Transposition of native chromatin for fast and sensitive epigenomic profiling of open chromatin, DNA-binding proteins and nucleosome position. Nat. Methods 10, 1213-1218 (2013).

45. Bolger, A. M., Lohse, M. \& Usadel, B. Trimmomatic: a flexible trimmer for Illumina sequence data. Bioinformatics 30, 2114-2120 (2014).

46. Li, H. \& Durbin, R. Fast and accurate short read alignment with Burrows-Wheeler transform. Bioinformatics 25, 1754-1760 (2009)

47. Zhang, Y. et al. Model-based analysis of ChIP-Seq (MACS). Genome Biol. 9 R137 (2008)

48. Quinlan, A. R. \& Hall, I. M. BEDTools: a flexible suite of utilities for comparing genomic features. Bioinformatics 26, 841-842 (2010).

49. Robinson, M. D. \& Oshlack, A. A scaling normalization method for differential expression analysis of RNA-seq data. Genome Biol. 11, R25 (2010)

50. Ewing, B., Hillier, L., Wendl, M. C. \& Green, P. Base-calling of automated sequencer traces usingPhred. I. Accuracy assessment. Genome Res. 8, 175-185 (1998).

51. Li, B. \& Dewey, C. N. RSEM: accurate transcript quantification from RNA-Seq data with or without a reference genome. BMC Bioinforma. 12, 1 (2011).

52. Leek, J. T., Johnson, W. E., Parker, H. S., Jaffe, A. E. \& Storey, J. D. The sva package for removing batch effects and other unwanted variation in highthroughput experiments. Bioinformatics 28, 882-883 (2012).

53. Qu, K. et al. Individuality and variation of personal regulomes in primary human T cells. Cell Syst. 1, 51-61 (2015).

54. Heinz, S. et al. Simple combinations of lineage-determining transcription factors prime cis-regulatory elements required for macrophage and B cell identities. Mol. Cell 38, 576-589 (2010).

55. Beekman, R. et al. The reference epigenome and regulatory chromatin landscape of chronic lymphocytic leukemia. Nat. Med. 24, 868 (2018)

56. Kelder, T. et al. WikiPathways: building research communities on biological pathways. Nucleic Acids Res. 40, D1301-D1307 (2012)

57. Wolf, F. A., Angerer, P. \& Theis, F. J. SCANPY: large-scale single-cell gene expression data analysis. Genome Biol. 19, 15 (2018).

58. Khan, A. et al. JASPAR 2018: update of the open-access database of transcription factor binding profiles and its web framework. Nucleic Acids Res. 46, D260-D266 (2017). 
59. Jolma, A. et al. DNA-dependent formation of transcription factor pairs alters their binding specificity. Nature 527, 384 (2015).

60. Bailey, T. L. et al. MEME SUITE: tools for motif discovery and searching Nucleic Acids Res. 37, W202-W208 (2009).

61. Chang, W., Cheng, J., Allaire, J., Xie, Y. \& McPherson, J. Shiny: web application framework for R. R package version 1, http://CRAN.R-project.org/ package $=$ shiny (2017).

62. Wickham, H. ggplot2: Elegant Graphics For Data Analysis. (Springer, 2016).

\section{Acknowledgements}

We thank Taneli Helenius for aid in scientific writing, research staff in the UConn Center on Aging for their help in recruitment and sample collection, JAX genomic technologies for their help with generating the sequencing data, and JAX Research IT for the support with building and maintaining the R Shiny application as well as with data upload to $\mathrm{dbGaP}$. We thank members of Ucar, Stitzel, Banchereau labs, Michael Stitzel, Karolina Palucka, Virginia Pascual, Derya Unutmaz for critical feedback during the progress of the study. We thank Yuqi Zhao and Magalie Collet for their help with dbGAP data upload. This study was made possible by generous financial support of the National Institute of General Medical Sciences (NIGMS) under award number GM124922 (to D.U.) and National Institutes of Health (NIH) grants R01 AG052608, R01 AI142086, UH2 AG056925 (to J.B.). Opinions, interpretations, conclusions, and recommendations are solely the responsibility of the authors and do not necessarily represent the official views of the National Institutes of Health (NIH). G.A.K is supported by the Travelers Chair in Geriatrics and Gerontology.

\section{Author contributions}

D.U., G.A.K., and J.B. designed the research. G.A.K. coordinated the clinical sample collection. C.C., R.M., and R.R. performed the experiments. E.M. pre-processed the sequencing data. E.M., D.U., D.J.M., D.N., and A.E. analyzed the data. E.M. and D.U. wrote the paper. D.J.M. implemented the R Shiny application. All authors revised the manuscript and helped with data interpretation.

\section{Competing interests}

The authors declare no competing interests.

\section{Additional information}

Supplementary information is available for this paper at https://doi.org/10.1038/s41467020-14396-9.

Correspondence and requests for materials should be addressed to D.U.

Peer review information Nature Communications thanks Jorg Goronzy and Ansuman Satpathy for their contributions to the peer review of this work.

Reprints and permission information is available at http://www.nature.com/reprints

Publisher's note Springer Nature remains neutral with regard to jurisdictional claims in published maps and institutional affiliations.

(c) (i) Open Access This article is licensed under a Creative Common Attribution 4.0 International License, which permits use, sharing, adaptation, distribution and reproduction in any medium or format, as long as you give appropriate credit to the original author(s) and the source, provide a link to the Creative Commons license, and indicate if changes were made. The images or other third party material in this article are included in the article's Creative Commons license, unless indicated otherwise in a credit line to the material. If material is not included in the article's Creative Commons license and your intended use is not permitted by statutory regulation or exceeds the permitted use, you will need to obtain permission directly from the copyright holder. To view a copy of this license, visit http://creativecommons.org/ licenses/by/4.0/.

(C) The Author(s) 2020 